to be Submitted to the Astrophysical Journal Supplement Series

\title{
An Online Catalog of Cataclysmic Variable Spectra from the Far
} Ultraviolet Spectroscopic Explorer

\author{
Patrick Godon ${ }^{1}$, Edward M. Sion \\ Astronomy E Astrophysics, Villanova University, \\ 800 Lancaster Avenue, Villanova, PA 19085, USA \\ patrick.godon@villanova.edu; edward.sion@villanova.edu \\ Karen Levay \\ Space Telescope Science Institute, Baltimore, MD 21218 \\ klevay@stsci.edu \\ Albert P. Linnell, Paula Szkody \\ Department of Astronomy, University of Washington, Box 351580, Seattle, WA 98195-1580 \\ linnell@astro.washington.edu ; szkody@astro.washington.edu \\ Paul E. Barrett \\ United States Naval Observatory, Washington, DC 20392 \\ barrett.paul@usno.navy.mil \\ Ivan Hubeny \\ Steward Observatory and Department of Astronomy and Astrophysics, University of \\ Arizona, Tucson, AZ 85721 \\ hubeny@as. arizona.edu \\ and \\ William P. Blair \\ Henry A. Rowland Department of Physics and Astronomy, The Johns Hopkins University, \\ Baltimore, MD 21218
}


wpb@pha.jhu.edu

\begin{abstract}
We present an online catalog containing spectra and supporting information for cataclysmic variables that have been observed with the Far Ultraviolet Spectroscopic Explorer (FUSE). For each object in the catalog we list some of the basic system parameters such as (RA,Dec), period, inclination, white dwarf mass, as well as information on the available FUSE spectra: data ID, observation date and time, and exposure time. In addition, we provide parameters needed for the analysis of the FUSE spectra such as the reddening $\mathrm{E}(\mathrm{B}-\mathrm{V})$, distance, and state (high, low, intermediate) of the system at the time it was observed. For some of these spectra we have carried out model fits to the continuum with synthetic stellar and/or disk spectra using the codes TLUSTY and SYNSPEC. We provide the parameters obtained from these model fits; this includes the white dwarf temperature, gravity, projected rotational velocity and elemental abundances of C, Si, S and N, together with the disk mass accretion rate, the resulting inclination and model-derived distance (when unknown). For each object one or more figures are provided (as gif files) with line identification and model fit(s) when available. The FUSE spectra as well as the synthetic spectra are directly available for download as ascii tables. References are provided for each object as well as for the model fits. In this article we present 36 objects, and additional ones will be added to the online catalog in the future. In addition to cataclysmic variables, we also include a few related objects, such as a wind accreting white dwarf, a pre-cataclysmic variable and some symbiotics.
\end{abstract}

Subject headings: accretion, accretion disks - novae, cataclysmic variables - white dwarfs

\title{
1. Introduction
}

This article presents the first version of an on-going online catalog of Far Ultraviolet Spectroscopic Explorer (FUSE; (Moos et al. 2000)) spectra of cataclysmic variables (CVs).

\footnotetext{
${ }^{1}$ Visiting at the Johns Hopkins University, Henry A. Rowland Department of Physics and Astronomy, Baltimore, MD 21218
} 
The catalog includes CV types and subtypes, as follows: dwarf novae (U Gem, Z Cam, SS Cyg, WZ Sge and SU UMa subtypes), nova-likes (VY Scl/anti-DN, UX UMa, SW Sex subtypes), and magnetic systems (IPs, Polars, DQ Her, AM Her subtypes). In addition the catalog also includes a few miscellaneous objects related to CVs such as novae (of all types), symbiotics, and pre-cataclysmic variables. In Table 1 we list the objects in the catalog as of March 2012. For each object the table includes RA (J2000), DEC (J2000), type and subtype, binary orbital period, system inclination, reddening value, white dwarf mass, and distance. For each object, there is (i) at least one FUSE spectrum, (ii) possibly a theoretical fit to the spectrum (when the continuum can be modeled successfully), and (iii) basic data about the system including figures and downloadable ascii tables. The state (outburst/high, quiescence/low or intermediate) of each system at the time of observation is specified on the object page (see sec.2.4).

While Froning et al. (2012) has presented a summary of FUSE spectra of cataclysmic variables, there are similarities and differences between their work and our catalog. The FUSE survey of Froning et al. (2012) is an atlas of all the existing FUSE spectra of all CVs, which includes basic information about each system (type, orbital period and inclination) and the FUSE exposures (data ID, exposure time, data quality, observation date and whether the data were obtained during a high/outburst or low/quiescent state). The spectra are discussed and characterized as a function of CV subtype: DNe in quiescence, which are divided into those that exhibit a flat continuum with emission lines and those exhibiting absorption lines (broad Lyman lines and narrower metal lines); DNe in outburst; nova-likes subdivided into narrow absorption lines, broad absorption lines, and with emission lines; intermediate polars; and polars. Intrinsic spectral lines as well as prominent ISM lines are tabulated. As such the paper of Froning et al. (2012) is really an Atlas of FUSE spectra of CVs, containing 99 objects where the spectra are catalogued according to their characteristics. The spectra are downloadable in fits format directly from the website (MAST) where a preview is presented including basic line identification (which is the same for all the spectra).

In comparison, our catalog is more oriented toward the modeling and analysis of the spectra. The FUSE spectra are downloadable as ascii tables which can then be easily used with computer modeling programs often used by theorists and/or compared directly with theoretical spectra obtained from (e.g.) TLUSTY and SYNSPEC (Hubeny 1988; Hubeny \& Lanz 1995). In addition to inclination and orbital period, our catalog also includes parameters that are usually needed in the modeling of spectra, such as white dwarf mass, distance, and reddening (when these are known). We include a preview of the spectrum spread over three panels for better viewing, with individual line identifications for each object separately, including ISM molecular hydrogen lines. When possible we provide results from the spectral modeling, including downloadable theoretical spectra in ascci format and 
additional system parameters such as mass accretion rate, WD temperature, stellar rotation rate, and chemical abundances. We also provide a list of references for each object. At the present time the catalog is still growing, and systems are being added with time. The catalog will eventually include all CVs and related objects that were observed with FUSE. In the near future the catalog will also include a large database of theoretical spectra of (solar composition) WDs covering a wide range of temperatures and gravity.

\section{Processing of the FUSE Spectra}

The FUSE telescope and its performance are described in detail by Moos et al. (2000); Sahnow et al. (2000), and we give here only a short overview of some of its features and characteristics, as needed to explain and justify some of the procedures we followed to extract the final spectra. Most of the FUSE data were obtained through the 30"x30" LWRS Large Square Aperture in TIME TAG mode, and were processed with the latest and final version of CalFUSE (v3.2.3; Dixon et al. (2007)). The FUSE data come in the form of eight spectral segments (SiC1a, SiC1b, SiC2a, SiC2b, LiF1a, LiF1b, LiF2a, and LiF2b) which are combined together to give the final FUSE spectrum. The spectral regions covered by the spectral channels overlap, and these overlap regions are then used to renormalize the spectra in the $\mathrm{SiC} 1, \mathrm{LiF} 2$, and $\mathrm{SiC} 2$ channels to the flux in the LiF1 channel. We then produce a final spectrum that covers the full FUSE wavelength range 905-1187 $\AA$.

The low sensitivity portions of each channel are usually discarded because of the low $\mathrm{S} / \mathrm{N}$ there. In most channels there exists a narrow dark stripe of decreased flux in the spectra running in the dispersion direction, known as the "worm", which can attenuate as much as $50 \%$ of the incident light in the affected portions of the spectrum; - this is due to shadows thrown by the repeller grid wires above the detector. Because of the temporal changes in the strength and position of the "worm", CalFUSE cannot correct target fluxes for its presence. Therefore, we carried out a visual inspection of the FUSE channels to locate the worm and we manually discarded that portion of the spectrum. CalFUSE, for the most part, takes care of removing several additional well-known issues, such as jitter and event bursts. For faint sources background subtraction can be a problem for CalFUSE and we did have to remediate this problem by either ignoring or discarding those portions of the spectra affected by bad background subtraction.

Due to these problems, for some of the systems, we had to discard the two $\mathrm{SiC}$ channels completely. For other systems, large portions of the $\mathrm{SiC}$ channels had to be removed and, as a consequence, some spectra exhibit a gap around $1080 \AA$. The channels that were the most reliable were the $1 \mathrm{aLiF}, 1 \mathrm{bLiF}$ and $2 \mathrm{aSiC}$. The $2 \mathrm{aLiF}$ channel was moderately reliable and 
was used especially when the worm was affecting the $1 \mathrm{bLiF}$ channel. The $1 \mathrm{aSiC}$ and $1 \mathrm{bSiC}$ channels were the most discarded, together with the $2 \mathrm{bLiF}$ channel. The $2 \mathrm{bSiC}$ channel was discarded only when it was found to be of much lower quality than the LiF channels.

In the final step, we combined the individual exposures (unless otherwise specified) and channels to create a time-averaged spectrum weighting the flux by the (good) exposure time and sensitivity of the input exposure and channel of origin, using a suite of FORTRAN programs, unix scripts and IRAF procedures (which were all writen specifically for this purpose).

The observation log for the initial objects included in the catalog is given in Table 2, where we list the FUSE data ID, the observation date, the observation time, the good exposure time and the state in which the system was observed (i.e. high, low or intermediate).

Details of the data processing for each individual system are provided in the reference(s) given in the link below the figure(s). Some of these details are also listed individually for each object in the "Description Panel" (see appendix B).

\section{The FUSE Spectral Lines}

The main emitting components contributing to the FUSE spectra of CVs are the accretion disk and the WD. Usually, the disk dominates during the high state (or outburst), while the WD dominates during the low state (or quiescence). The main feature of the spectra is the broad Ly $\beta$ absorption feature, which can easily be used to assess the gravity and temperature of the emitting gas (when this feature is not affected by e.g. large rotational broadening, strong O VI emission/absorption lines, and so on). At higher temperatures, as the continuum rises in the shorter wavelengths, the higher orders of the Lyman series also become visible and can also be used (when possible) to accurately assess the temperature and gravity of the emitting gas.

Additional broad absorption lines of metals (C, S, Si, ..) are detected and help determine the chemical abundances and projected rotational velocity of the emitting gas. In the present spectra, the main absorption features observed are: C II (1010 $)$, C III (1175 ̊), Si III (1108-

$1114 \AA$ and $1140-1144 \AA)$, Si IV (1067 $\AA$ and 1120-1130 $)$, S IV (1073 $\AA$ ), and N II (1085 when not contaminated by air glow).

On top of the spectrum, broad emission lines are also found in some of the systems, mainly the O vi doublet and C III (977 $\AA$ and $1175 \AA$ ), indicative of wind activity.

In Table 3 we provide a list of the ions and spectral lines expected for these sources in 
the FUSE spectral range. The wavelengths are the theoretical rest wavelengths in $\AA$.

For al the systems we assign four line identification quality values as follows.

A - a fully reliable line identification without contamination, the central wavelength of the line can easily be determined.

B - a reliable line identification with possible contamination or/and blended with another line, the central wavelength of the line cannot be determined with precision.

$\mathrm{C}$ - a possible line identification, but less reliable than $\mathrm{B}$, due to low $\mathrm{S} / \mathrm{N}$ and other factors. A spectral feature is seen but is not identified with certainty.

D - the line is either not detected, or the detection is not reliable at all; the line identification on the figure is tentative or is only informative (to better show that the line is missing where it should be). A very noisy spectral feature is seen, or no spectral feature is detected at all (flat continuum).

For all the systems, in Table 4 we tabulate these quality values for the most common spectral lines seen in CVs.

Most of the FUSE spectra show some ISM molecular hydrogen absorption. The most affected targets reveal a spectrum literally "sliced" at almost equal intervals $(\sim 12 \AA)$; starting at wavelengths around $1110 \AA$ and continuing towards shorter wavelengths all the way down to the hydrogen cut-off around $915 \AA$. In the affected FUSE spectra, we identified the most prominent molecular hydrogen absorption lines by their band (Werner or Lyman), upper vibrational level (1-16), and rotational transition ( $\mathrm{R}, \mathrm{P}$, or $\mathrm{Q}$ ) with lower rotational state $(\mathrm{J}=1,2,3)$. The most common ISM lines are tabulated in Table 5.

In addition, the targets that are weak FUSE sources exhibit sharp emission lines from air glow (geo- and helio-coronal in origin; some of which are due to sunlight directly reflected

inside the telescope), such as the H I series, S vi (934 $\AA$ and $944 \AA$ ), Ovi doublet, CiII $(977 \AA)$, and He I $(1168 \AA)$.

For each system, we mark on the (online) figures the lines that are detected as well as lines that are commonly seen for comparison.

\section{Spectral Modeling}

\subsection{The Synthetic Stellar Spectral Codes}

We created model spectra for high-gravity stellar atmospheres using the codes TLUSTY and SYNSPEC (http://nova.astro.umd.edu; Hubeny (1988); Hubeny \& Lanz (1995)).

Atmospheric structure is computed (using TLUSTY) assuming either a LTE or a NLTE 
atmosphere for a given stellar surface temperature and gravity, where the switch to NLTE is made for hot models $(T>30,000 \mathrm{~K})$. In that manner, we generate photospheric models with effective temperatures ranging from $12,000 \mathrm{~K}$ to $75,000 \mathrm{~K}$ in increments of about $10 \%$ (e.g. 1,000 K for $\mathrm{T} \sim 15,000 \mathrm{~K}$ and 5,000 K for $\mathrm{T} \sim 70,000 \mathrm{~K}$ ). We choose values of the surface gravity $\log (g)$ ranging between 7.0 and 9.5. The (converged) output stellar atmosphere model is then used as an input for the synthetic spectrum generator code SYNSPEC, and finally the code ROTIN is used to simulate the broadening of the lines due to stellar rotation and/or instrumentation. Limb darkening is also included in ROTIN. The stellar rotational velocity $V_{\text {rot }} \sin (i)$ varies from a few $\mathrm{km} \mathrm{s}^{-1}$ to $1000 \mathrm{~km} \mathrm{~s}$. In order to fit the absorption features of the spectrum, we also vary the chemical abundances of the main species C, N, S and Si. For any WD mass, there is a corresponding radius, or equivalently, one single value of $\log (g)$ Hamada \& Salpeter 1961), which also depends on the temperature and composition of the WD (Wood 1995; Panei et al. 2000).

The same suite of codes can also be used to generate spectra of accretion disks (Wade \& Hubeny 1998) based on the standard alpha disk model (Shakura \& Sunvaev 1973). The standard disk model assumes a geometrically thin axi-symmetric disk, one-dimensional, with a nearly Keplerian profile in which the viscosity is due to MHD turbulence. The density and temperature profiles are function of $r$, and the energy dissipated due to the shear between adjacent rings of matter is radiated locally in the vertical direction $(z)$. The disk model consists in dividing the disk into $\mathrm{N}$ rings of radii $r_{i}(i=1,2, . . N)$, where the temperature $T\left(r_{i}\right)$ and density $\rho\left(r_{i}\right)$ of each ring are given by the standard disk model. The TLUSTY \& SYNSPEC codes are then used to generate spectra for the disk rings, given the white dwarf mass, mass accretion rate, and radius of the ring. The spectra of the rings are then added together to generate a disk spectrum for a given inclination and assuming limb darkening. In the present work we use disk spectra from the grid of solar composition disk spectra generated by Wade \& Hubeny (1998) (assuming LTE only) as well as disk spectra that we generated using both the LTE and NLTE options for disk rings, as well as in some cases varying the chemical abundances. A detailed description of the procedure to generate such disk spectra is given by Wade \& Hubeny (1998). Composite models of WD+disk take into account the geometry of the system and account for the visibility of the WD.

\subsection{Modeling the ISM Hydrogen Absorption Lines}

For most systems showing ISM atomic and molecular hydrogen absorption lines, we

identify these lines in the figures to avoid confusing them with the WD lines. For some systems, however, ISM lines are deep and broad and some of the WD lines (such as SIV 
(1062.6 $\AA$ \& $1073 \AA$ ) are located at almost the same wavelengths as some of the ISM lines. For that reason, we model the ISM lines; this helps us not only to differentiate between the WD lines and the ISM lines, but it improves the overall spectral fit, and provides us with the atomic and molecular column densities.

The ISM spectra models are generated using a program developed by one of us (PEB). This program uses a custom spectral fitting package to estimate the temperature and density of the interstellar absorption lines of atomic and molecular hydrogen. The ISM model assumes that the temperature, bulk velocity, and turbulent velocity of the medium are the same for all atomic and molecular species, whereas the densities of atomic and molecular hydrogen, and the ratios of deuterium to hydrogen and metals (including helium) to hydrogen can be adjusted independently. The model uses atomic data of Morton (2000, 2003) and molecular data of Abgrall et al. (2000). The optical depth calculations of molecular hydrogen have been checked against those of Morton (2003). The ratios of metals to hydrogen and deuterium to hydrogen are fixed at 0 and $2 \times 10^{-5}$, respectively, because of the low signal-to-noise ratio data. The wings of the atomic lines are used to estimate the density of atomic hydrogen and the depth of the unsaturated molecular lines for molecular hydrogen. The temperature and turbulent velocity of the medium are primarily determined from the lines of molecular hydrogen when the ISM temperatures are $<250 \mathrm{~K}$.

The ISM absorption features are best modeled and displayed when the theoretical ISM model (transmission values) is combined with a synthetic spectrum for the object (namely a WD synthetic spectrum, a disk spectrum or a combination of both).

\subsection{Synthetic Spectral Model Fitting}

Before carrying out a synthetic spectral fit, we masked portions of the spectra with strong emission lines, strong ISM molecular absorption lines, detector noise and air glow. These regions of the spectra are somewhat different for each object and are not included in the fitting. The regions excluded from the fit are in blue in the figures. The excluded ISM quasi-molecular absorption lines are marked with vertical labels in the figures.

After having generated grids of models for each target, we use FIT, a $\chi^{2}$ minimization routine (see e.g. Press et al. (1992)), to compute the reduced $\chi_{\nu}^{2}\left(\chi^{2}\right.$ per number of degrees of freedom $\nu$ ) and scale factor values for each model fit. While we use a $\chi^{2}$ minimization technique, we do not blindly select the least $\chi^{2}$ models, but we examine the models that best fit some of the features such as absorption lines and, when possible, the slope of the wings of the broad Lyman absorption features. When possible, we also select the models that are 
in agreement with the distance of the system (or its best estimate).

The flux level at $1000 \AA$ (between $\operatorname{Ly} \beta$ and $\mathrm{Ly} \gamma$ ) is close to zero for temperatures below $18,000 \mathrm{~K}$; at $30,000 \mathrm{~K}$ it is about $50 \%$ of the continuum level at $1100 \AA$ and it reaches $100 \%$ for $T>45,000 \mathrm{~K}$. At higher temperature $(T>50,000 \mathrm{~K})$ the spectrum becomes fairly flat and there is not much difference in the shape of the spectrum between (say) a 50,000 K and a $80,000 \mathrm{~K}$ model. When fitting the shape of the spectrum in such a manner, an accuracy of about $500-1,000 \mathrm{~K}$ is obtained, due to the $\mathrm{S} / \mathrm{N}$. In theory, a fine tuning of the temperature (say to an accuracy of about $50 \mathrm{~K}$ ) can be carried out by fitting the flux levels such that the distance to the system (if known) is matched. However, the fitting to the distance depends strongly on the radius (and therefore the mass) of the WD. In most of the systems the mass of the WD and the distance are unknown and therefore the temperature of a system is often accurate to only $\sim 500-1000 \mathrm{~K}$. Furthermore, since the Ly $\beta$ profile depends on both the temperature and gravity of the WD, the accuracy of the solution is sometimes further decreased as there is a small degeneracy in the solution, namely the solution spreads over a small region of the $\log (g)$ and $T$ parameter space. And last, reddening values are not always known, therefore increasing even more the uncertainty in assessing the temperature by scaling the synthetic flux to the observed flux. As a consequence, for some targets we may present more than one model fit.

The WD projected rotational velocity $\left(V_{\text {rot }} \sin (i)\right)$ is determined by fitting the WD model to the spectrum while paying careful attention to the line profiles. We did not carry out separate fits to individual lines but rather tried to fit the lines and continuum in the same fit while paying careful attention to the absorption lines.

For most spectra, when possible, we tried to fit a single WD model, a single disk model, and a composite WD+disk model, assuming different reddening values. Systems that were observed mainly in the low state can usually be modeled just with a WD spectrum, while systems observed in a high state usually require the addition of a disk model. In systems for which the WD mass (and radius) is unknown, the distance is estimated using the maximum magnitude/orbital period relation (Warner 1995; Harrison et al. 2004) or, when the period is unknown, we estimate the distance using the secondary infrared emission technique of Knigge (2006, 2007).

For the single WD model, we vary the temperature while first keeping the WD mass constant, starting at about $0.4 M_{\odot}$. Once the lowest $\chi^{2}$ has been found for a given mass, we vary the projected rotational velocity, and possibly also the abundances, to further lower the $\chi^{2}$ and obtain a best fit. Once the best fit has been found for that mass, we assume a slightly larger mass and again vary the temperature until the lowest $\chi^{2}$ is found. We follow this procedure iteratively until we reach a mass of about $1.2 M_{\odot}$. The next step is to find, 
from all these lowest $\chi^{2}$ models, the one which agrees best with the distance estimate, or the one which has the lowest $\chi^{2}$ of all (if the constraint on the distance cannot be used). For the single disk model, we carry out a similar procedure by varying the mass accretion rate and inclination assuming discrete values of the WD mass, and then choose the least $\chi^{2}$ model agreeing best with the distance. We use a similar procedure for the WD+disk composite modeling to find the best fit model.

In Table 6 we list the model fits we carried out for a number of systems. These appear in the same manner as in the catalog. In the next section we concentrate on a few systems as examples to illustrate the diversity of model fits.

\section{Examples}

We give here a few examples of systems exhibiting model fits with increasing level of complexity. For the details of the model fits of all the systems in Table 6, the reader is referred to the references in that Table.

The simplest and easiest objects to model are those in which the WD is the sole (or dominant) component at very low accretion rates. In that case the spectrum resembles that of a field WD, with little or no metal lines and with a low stellar rotational rate. This is the case for the pre-CV (a post-common envelope binary) V471 Tau depicted in Figure 1. This eclipsing system has a magnetic WD accreting from the poles at a low accretion rate. The continuum flux is characterized mainly by the absorption features of the Lyman series onto which are superposed a few shallow metal absorption lines. The FUSE spectrum of V471 Tau was obtained as part of a Periodic Channel Coalignment program (rather than a "usual" scientific research GO program) and, as a consequence, the spectrum had to be binned in time in a very specific manner to extract the data obtained only during the good exposure time (this is described in detail in the next section). This FUSE spectrum has not been modeled previously. The results of the model fit to the FUSE spectrum are completely consistent with the results obtained from fitting the STIS spectrum of the system at longer wavelengths (Sion et al. 2012), namely, the WD has a gravity $\log (g)=8.3$, a temperature $T_{w d}=33,400 \mathrm{~K}$, sub-solar abundances (of the order of $1 \%$ solar and lower) and a broadening of the absorption lines corresponding to $250 \mathrm{~km} \mathrm{~s}^{-1}$, about a factor of 4 larger than expected, a possible indication that the broadening is due to a weak Zeeman effect.

In all the online figures, the flux (vertical axis) is given in $\operatorname{ergs~} \sec ^{-1} \mathrm{~cm}^{-2} \AA^{-1}$ and the wavelength (horizontal axis) is given in Angströms $(\AA)$. For clarity all the spectra are shown on three panels: the upper panel from about $900 \AA$ to $1000 \AA$, the middle panel from about 
$1000 \AA$ to $1100 \AA$, and the lower panel from about $1100 \AA$ to $1200 \AA$. Air glow emission is annotated above each panel with a "+" sign inside a circle. Strong emission lines reaching the upper part of the frame in each panel are marked just above the panel. The Hydrogen series is marked below the x-axis in the upper and middle panel and the hydrogen cut-off limit is clearly marked around $915 \AA$ with an increasing density of tick marks. The He I (1168 $\AA$ ) line is marked below the lower panel. When a model is shown, the portions of the observed spectrum that have been masked before the spectral fit are in blue, while the rest of the observed spectrum is in red. The model fit is shown with a solid black line (on a white background) or a solid white line (on a black background).

As the mass accretion rate increases, the WD spectrum becomes more affected by the metal lines. This is demonstrated in Figures 2 and 3. Figure 2 depicts the FUSE spectrum of the wind accreting WD P831-57. The model fit has a WD with $\log (g)=7.85, T_{w d}=$ $37,500 \mathrm{~K}$, with $1-2 \%$ solar abundances and a slow rotational velocity. The details of this model fit are given in Barrett et al. (2012). In Figure 3 we show the spectrum of the novalike MV Lyr obtained in its low state, fitted with a solar abundance WD model. The metal lines are much broader and deeper than in Figures $1 \& 2$. The temperature of the WD reaches 45,000 K. One can clearly see, from Figures 1-3, how the flux steadily increases in the shorter wavelengths of FUSE as the WD temperature increases. Some cool WD models include the effect of quasi-molecular HI absorption features, in the catalog these are labeled "Q-mol" in the appropriate Figures.

Not all the systems exhibit spectra that can be easily modeled as emission lines (from the sources, or geo- helio- coronal in origin) and ISM absorption lines can also impact the spectrum. We show such a spectrum in Figure 4 for the symbiotic binary AE Ara. In the figures in the online catalog, neutral oxygen lines (when present) are shown with an arrow "OI $->$ "; if many (ISM) OI lines are present, they are just annotated with vertical tick marks in the lower portion of each panel. ISM molecular hydrogen lines are either individually annotated vertically (e.g. L10R1, as in Figure 4) or just marked with an arrow "MH ->" and vertical tick marks (usually in the upper part of each panel). Features resulting from fixed pattern noise introduced by FUSE detectors are indicated with "FPN".

As a more complex example, we choose the FUSE spectrum of the dwarf nova (Z Cam) system EM Cyg, which is shown in Figure 5. The data were obtained in quiescence and consists of four exposures taken at different binary orbital phase. We modeled the exposure obtained when absorption was minimal, corresponding to the WD facing the observer. The $\mathrm{S} / \mathrm{N}$ of a single exposure is rather low and the spectrum is also affected by ISM absorption. This system is dominated by emission from the WD but a best fit is obtained with the inclusion of a low mass accretion disk model (see Figure 5) and ISM molecular absorption 
curtain. In the catalog, as in Figure 5, the disk model and the WD model are shown respectively with dashed- and dotted lines.

As a last example, we choose the UX UMa nova-like variable V3885 Sgr observed in a high state, shown in Figure 6. This FUSE spectrum is different than the ones shown in the previous figures, and it is characteristic of systems accreting at a high accretion rate. The spectrum is characterized by a continuum flux extending to the shortest wavelengths of FUSE, where the Lyman series absorption features are not as obvious as in the previous spectra due mainly to Keplerian rotational broadening. In addition, broad, deep, and blueshifted absorption lines of highly ionized species (such as NIV, S vi, O vi, .. ; see Figure 6 ), are indicative of a hot corona forming above the disk or boundary layer(BL). The sharp absorption lines of H I are of interstellar origin. Systems in high states are modeled mainly with an accretion disk with a large $\dot{M}$ including the contribution from a hot WD, which usually does not contribute much to the overall flux. Here we improve the disk model, by including the contribution of the BL to the theoretical spectra. We replace the inner disk rings with hot rings matching the temperature of the $\mathrm{BL} \sim 10^{5} \mathrm{~K}$. We built different $\mathrm{BL}$ models, consisting of $1,2,3$ or more rings assuming different ring temperatures ranging between $100,000 \mathrm{~K}$ and $200,000 \mathrm{~K}$. We generated models consisting of all the possible combinations of $\mathrm{BL}+$ disk $+\mathrm{WD}$, over a wide range of parameters keeping the assumption the BL luminosity should not exceed the disk luminosity. We then apply our least $\chi^{2}$ fitting routine after we masked all the sharp ISM lines and the broad emission lines which are all blue-shifted by $\sim 2 \AA$ (an indication that they form in a corona above the emitting component). The final (best) model is found based not only on the the least $\chi^{2}$ value, but also by checking which (least $\chi^{2}$ ) model agrees best with the distance, white dwarf mass, inclination, and mass accretion rate (if and when these are known). The best model to the FUSE spectrum of V3885 Sgr consists of a $0.7 M_{\odot}$ WD with a temperature of $60,000 \mathrm{~K}$ WD, $200 \mathrm{~km} / \mathrm{s}$, a disk inclined at $60^{\circ}$ with $\dot{M}=3 \times 10^{-9} M_{\odot} / \mathrm{yr}$, and a BL with a temperature of $175,000 \mathrm{~K}$ (see Figure 6).

We note that a hot WD and a high $\dot{M}$ disk also provide a satisfactory fit (Linnell et al. 2009), but in order to match the flux in the shorter wavelengths of FUSE one needs to include the boundary layer.

We are now implementing disk models with the inclusion of a realistic boundary layer to improve the modeling of such systems in the high state. In the FUSE spectral range, one has to include mainly the hottest components of the system, namely the WD, the boundary (or spread) layer, and the accretion disk. 


\section{Acknowledgements}

PG is pleased to thank the Henry Augustus Rowland Department of Physics and Astronomy at the Johns Hopkins University, Baltimore, MD, for hospitality. This work was supported by the National Aeronautics and Space Administration (NASA) under grant number NNX08AJ39G issued through the Office of Astrophysics Data Analysis Program (ADP) to Villanova University. We have used some of the online data from the AAVSO, and are thankful to the AAVSO and its members worldwide for making this data public and for their constant monitoring of cataclysmic variables. This work is based on observations made with the NASA-CNES-CSA Far Ultraviolet Spectroscopic Explorer. FUSE was operated for NASA by the Johns Hopkins University under NASA contract NAS5-32985.

\section{A. Catalog Description}

The FUSE catalog is maintained at the Mikulski Archive for Space Telescopes (MAST) as a High-Level Science Product (HLSP). There is a search interface where users can specify query parameters to find objects of interest. From the search results, the user can bring up a webpage for each target that contains figures, downloadable data, system parameters and model parameters. The catalog data are stored in a relational database and the image components for the web pages are stored online. The webpages for each object are built dynamically using PHP scripts using data retrieved from the database and the online images. This permits the catalog to easily accommodate growth, enhancements, and if necessary, corrections. The FUSE mission search interface results also include links leading to the catalog thus providing general MAST users easy access. The catalog is found at the URL: http://archive.stsci.edu/prepds/cvaro/

\section{A.1. Definitions}

The target name is the name of the system as found in the MAST database. For most

of the systems it is the name as given in the Ritter \& Kolb (2003) Catalog of CVs (the most common objects). For less common objects not included in the Ritter \& Kolb Catalog of CVs, it is the name as given in the Downes et al. (2001) Catalog of CVs. When applicable we also give the additional name(s) of the object. For example, WD 0334-6400 is a DA+dMe binary in which the WD is undergoing accretion of the companion star's wind. It is not a CV though it was classified as one in the Downes et al. (2001) catalog. WD 0334-6400 is also known as Ret 1 or P831-57, and it is listed in our catalog under all three names. 
Type: in the catalog we define four types of Catalysmic Variables as follows:

DN: Dwarf Nova;

NL: Nova-Like;

Mg: Magnetic Systems;

Msc: Miscellaneous variables.

If only the type is entered (without any other selection), the search returns all the objects classified under this type.

Subtype: the objects in the catalog are further divided into subtypes as follows.

DN subtype:

UG - U Gem;

Z - Z Cam;

SS - SS Cyg;

WZ - WZ Sge;

SU - SU UMa.

NL subtype:

VY - VY Scl (anti-DN);

UX - UX UMa;

SW - SW Sex.

Mg subtype:

IP - Intermediate Polar (with a moderately strong magnetic field; disrupted inner disk);

P - Polar (with a strong magnetic field; devoid of disk);

DQ - DQ Her;

AM - AM Her.

Msc subtype:

$\mathrm{N}$ - Nova (all types of novae);

CV - all other type/subtype of Cataclysmic Variables;

$\mathrm{R}$ - related to CVs: Symbiotics;

PCV - Pre Cataclysmic Variable.

If only the subtype is entered (without any other selection), the search returns all the objects classified under this subtype.

$P$ is the orbital period of the binary (if known) given in hr.

$i$ is the inclination of the system (if known) and is given in degrees. It is also the 
inclination used in the modeling of the synthetic disk spectrum to fit the observed spectrum (it ranges between 0 and 90).

$E(B-V)$ is the reddening (extinction) toward the object. The spectrum was dereddened assuming this value prior to the spectral fit. Unless otherwise specified, the reddening value is taken for each system from the works of Verbunt (1987); La Dous (1991); Bruch \& Engel (1994).

$M_{w d}$ is the mass of the WD of the system given in units of solar mass. This mass is usually taken from the literature and the entry is left blank if it is unknown. In the modeling we use the $\log$ of the surface gravity $(\log (g))$ rather than the mass of the WD; it corresponds to the effective surface gravity (acceleration in $\log$ and cgs units) of the WD used to generate the stellar atmosphere or accretion disk model to fit the observed spectrum (it ranges between 7.0 and 9.5).

$T_{w d}$ is the effective temperature of the WD, given in Kelvin, that was used to generate the stellar atmosphere model to fit to the observed spectrum (usually between 10,000 K and $100,000 \mathrm{~K})$.

$V_{\text {rot }} \sin i$ (online as Rotv) is the projected rotational velocity of the WD (in $\mathrm{km} \mathrm{s}^{-1}$ ) used to generate the line broadening of the stellar atmosphere model in the fit to the observed spectrum, it usually ranges between $10 \mathrm{~s} \mathrm{~km} \mathrm{~s}^{-1}$ to a few $100 \mathrm{~km} s^{-1}$.

$d$ is the distance to the object given in parsecs and it is the distance used in the spectral modeling to fit the observed spectrum. If the distance is not known from the literature, then the work of Knigge (2006, 2007) is used to gain information on the secondaries and to estimate a lower limit on the distance.

$C$ is the abundance of carbon in solar units as derived from spectral modeling.

$S i$ is the abundance of silicon in solar units as derived from spectral modeling.

$S$ is the abundance of sulfur in solar units as derived from spectral modeling.

$N$ is the abundance of nitrogen in solar units as derived from spectral modeling.

Abundances are the abundances of all the species: either solar, or non-solar or as specified (e.g. $\mathrm{N} / \mathrm{C}>10)$.

The mass accretion rate is given in the modeling in $\log$ units of solar masses per year. This is the mass accretion rate that was used in the spectral disk modeling to fit the observed spectrum, and it usually ranges anywhere between -12.0 and -8.0. 


\section{A.2. Search Page}

One can either search for an object using the usual search keys (such as Target Name, RA \& Dec, ..), or by Type and Subtype (returns all objects listed in that category). One can also use the User-specified fields $1 \& 2$ as follows. Choose the User-specified field 1, e.g. $T_{w d}$ for the WD temperature, and enter in the Field Description the lower limit for Twd in Kelvin, e.g. '>19999' (without the quotation mark and enter the thousands without a coma); do the same with the User-specified field 2 for the upper limit for Twd, e.g. '<30001'. Hit search and all the systems with a WD temperature between 20,000 K and 30,000 K are returned. The temperature returned here is the one obtained from the modeling or known from the literature. If the search button is hit without filling in any key, the entire list of objects is returned. Note: the other buttons (e.g. maximum records, records per page,...) are the ones usually found in any search on MAST. The object(s) from the search is(are) returned in a table ("Search Results Page").

\section{A.3. Search Results Page}

The Search Results Page returns the objects (Targets) as a table. The table contains from left to right:

- Mark: this button marks the dataset for which all the available files (spectrum, model and figure) can be downloaded by pressing the "Download marked data" button (use this button only if you want more than just the FUSE spectrum).

- Target Name. By clicking on the name of the target the "Object Page" is returned. This is the most important link for each object!

- RA and Dec (in Julian 2000).

- Type.

- Subtype.

- The Period.

- The inclination.

- The reddening.

- The WD mass.

- The WD effective surface temperature.

- The projected rotational velocity.

- The abundances of Carbon (C), Silicon (Si), Sulfur (S), Nitrogen (N) and Abundances in general.

- The distance.

- Data ID in last column is just a key for the software (no use for the user). 
In Table 1 we show a table similar to the search results page, in which we have omitted the WD temperature, projected rotational velocity and abundances.

\section{A.4. Object Page}

The object page displays all the information, data and graphics for an individual object (system). This is that page you will want to access when you are interested in a particular object. The Object Page is obtained (or 'returned', or 'accessed') by clicking directly on the name of an object in the Search Results Page table. The object page is divided into several panels.

The Description Panel. The upper panel is the "description" panel, with the name of the object in bold. On the left, it contains the basic parameters for that object: RA, Dec, Type, Subtype, $P, i, \mathrm{E}(\mathrm{B}-\mathrm{V})$. On the right, this upper panel displays a basic description

of the FUSE spectrum/spectra, the modeling/s and the figure/s displayed below. In the bottom of the upper panel there is a direct link to a list of references ("bibliography") for that object. That list is not complete as it only contains the most important references and the references relevant to the FUSE spectra and its modeling for that object. Do not hesitate to contact us to suggest additional references.

The Figure(s). For most objects there is only one additional panel located under the upper (description) panel. In that panel, on the right, there is a figure (in GIF format) displaying the FUSE spectrum (in red) of the object together with a synthetic (model) spectrum (in white or black). Line identification is included in the figure (for a description of the lines see "The FUSE Spectral Lines" below). By clicking on the figure one can view the figure by itself, and by clicking on it again a full resolution of the figure is returned (enlarged). In most cases the features in the spectrum that are not associated with the source (e.g. ISM lines, geocoronal emission, etc..) are marked in blue and masked before the modeling is carried out. Under the figure is listed the reference in which the original data analysis and modeling were carried out.

Observation Log Display. On the left to the figure, under the title FUSE Data, information on the FUSE spectrum is given (DataID, Observation Date and Time, good exposure time, state in which the system was observed: HIGH/OUTBURST, LOW/QUIESCENCE, or INTERMEDIATE) together with a direct link ("Download FUSE data") to the FUSE spectrum in ascii format (a table with wavelength, flux and error; the table contains a header with basic info; the units are as shown in the figure). 
Model Fit Display. Below this, under the title Synthetic Spectrum, the parameters that were used to model the spectrum are given, e.g. whether it is a simple disk model (DISK), or a WD, or a combination of both (WD+Disk), together with the quantities used (inclination, reddening $\mathrm{E}(\mathrm{B}-\mathrm{V})$, gravity $\log (g)$, temperature $(\mathrm{K})$, projected rotational velocity $\left(\mathrm{km} \mathrm{s}^{-1}\right)$ of the WD, C, Si, S and N abundances in solar abundances, distance in pc, mass accretion rate $\left(\log (\dot{M})\right.$ in $\left.M_{\odot} / \mathrm{yr}\right)$. Below that is a direct link to the model ("Download Synthetic Spectrum") which is given in ascii format as a table with wavelength and flux. The ascii table of the model contains a short header with basic information and the units are as shown in the figure.

Some systems were not modeled and do not have a synthetic spectrum and, therefore, that part under "Synthetic Spectrum" has no data.

Some systems have more than one FUSE spectrum and this is reflected by having more than one data block under the title FUSE data and/or (possibly) more than one figure.

Some systems have more than one model and this is reflected by having more than one data block under the title Synthetic Spectrum and/or more than one figure.

Notes On Figures. The bottom panel in the Object Page contains general notes on the figures. These notes can be found here in section 5 .

\section{References for Individual Objects}

We list references for each object in Table 7 . In the online catalog, references for each object are given in the upper part of the object page under the link "Bibliography". This does not cover all the existing references for the object as some objects have been extensively studied. If you find that an important reference is missing, please contact us ( patrick.godon@villanova.edu ) and we will incorporate any additional relevant reference you provide. Do not hesitate to contact us for comments, error and suggestions.

For the IP systems, instead of providing a list of references, we provide for each IP a direct link to the system in the online catalog of IPs of Koji Muka:1.

For each system, a link is also given under each figure to the work in which the data analysis was originally carried out. That reference usually contains the full details of the data processing and modeling and is listed here for each system separately in Appendix B.

\footnotetext{
${ }^{1}$ http://asd.gsfc.nasa.gov/Koji.Mukai/iphome/iphome.html (this is a case sensitive URL).
} 


\section{B. Individual Objects}

In this section, we provide notes on individual objects that are included in the initial release of the online catalog. The search function in the online catalog returns the objects listed in alphabetical order, and for this reason we list here the objects in alphabetical order.

AE Aquarii The spectrum of the Intermediate Polar AE Aqr shows broad and strong emission lines of N III + HeII ( 990), O vi (doublet), and He II (1085). We note, however, the absence of the usually strong C III (977 \& 1175) emission lines; instead, we tentatively identify these lines with the weak spectral features there. Weak broad emission lines of S IV (1063 \& 1073) and N IV (923) are detected. The S VI (933 \& 944) emission lines which are sometime seen in systems exhibiting a variety of rich emission lines (e.g. such as e.g. DW UMa (Hoard et al. 2003)) are not detected at all but they are marked on the figure. The FUSE exposures cover the entire orbital phase and the spectrum was obtained by combining the exposures obtained when the emission from the poles is minimal. However, the contribution from the white dwarf produces only a very weak continuum, while the matter shocked at the poles emits the broad and strong emission lines. This object exhibits the N/C anomaly indicative of CNO processing, as first noted in studies at longer UV wavelengths (Gänsicke et al. 2003).

AE Arae The FUSE spectrum of the Symbiotic AE Ara (Figure 4) shows many ISM absorption lines, in particular the molecular hydrogen lines. The very sharp emission lines of the Hydrogen Lyman series and OI are most probably geo- and heliocoronal in origin. The sharp C III (1175), S vi, S IV lines are from the source, though some of the sharp lines might be contaminated with sunlight reflected in the SiC channels (e.g. C III 977). We note that the S VI $(933,944)$ and the oxygen doublet appear to have two components: the first is in emission at about the rest wavelength, and the second is in absorption with a blue shift of at least $2 \AA$.

AG Draconis The spectrum of the Symbiotic AG Dra is characterized by sharp emission lines from highly ionized elements from the nebula being ionized by the hot component(s) of the system. These lines include Nev, Nevi, NeviI lines as well as lines from the He II Balmer series. S IV, S VI sharp emission lines are also seen as well as lines of Fe II (1141.17) and Fe III (1142.43) fluoresced by the very broad O VI (sharp O VI emission lines are also present). The ISM absorption lines include Si II, FeII, NI and Ar I lines. The rest of the continuum is rather flat indicative of a very hot component. 
AM Cassiopeiae AM Cas was observed with FUSE in an intermediate state of brightness. The longer wavelengths of the spectrum are strongly affected by the worm. The spectrum was modeled with $\mathrm{WD}+$ disk model assuming both $\mathrm{E}(\mathrm{B}-\mathrm{V})=0$ and $\mathrm{E}(\mathrm{B}-\mathrm{V})=0.2$. The distance to the system is possibly around $350 \mathrm{pc}$. The mass of the WD is unknown and so is the system inclination. We assessed $M_{w d}$ and $i$ here from the modeling. Note the difference between model 1 and model 2 in Table 6. Details on the processing of the observed spectrum and the spectral analysis are given in Godon et al. (2009a). The two models shown in the Figures (online) do fit the longer wavelengths of the FUSE spectrum but in the shorter wavelengths the observed flux is larger than in the models. The excess of flux could be due, for example, to broad emission from N IV $(923)$ and S vi $(933,944)$ but as shown in the figures the observed features are not at the expected wavelengths and do not have the proper line profile, in addition excess of flux also occurs around 950 to $960 \AA$, and the line notation is therefore only suggestive. Lines such as Si III (1110) and Si IV (1120-1130) make large shallow depression which fit the disk-dominated model, indicative that Keplerian broadening is responsible for their appearence. The ISM absorption lines (molecular hydrogen) are colored blue in the Figures so as to not be confused with the other lines.

AM Herculis The FUSE spectrum of the Polar AM Her, obtained in its low state, consists of 29 individual exposures, giving a complete coverage of the orbital phase of the binary (from 0 to 1 ). There are some sharp but weak ISM molecular hydrogen absorption lines as well as some metals: Fe II, Ar I, N II, C II and P II. The O vi lines are possibly due to airglow. We mark the position of the C III (1175) line to emphasize its absence. The WD is clearly visible and is the main source of FUV light. The combined spectrum is available for download as well as the individual exposures (1 through 29).

AQ Mensae Due to their contamination with air glow and very low $\mathrm{S} / \mathrm{N}$, the LiF 1 and $\mathrm{SiC} 1$ \& 2 channels have been discarded. The system is always at about the same magnitude (14-15). Details on the processing of the observed spectrum are given in Godon et al. (2009a). The S/N is so low that one cannot identify lines. However, some of the usually most prominent ISM molecular hydrogen absorption lines coincide with some low flux regions in the spectrum, indicating that the features might be real (e.g. L5R0, L5R1, L5R2, L4R0, L4R1, L4P1, L4R2, .. through L0R0, L0R1, L0P1). There is also a spectral feature that could be associated with the CIII (1175) line, though the overal flux level in that region is not reliable. Lines have been marked in the online Figures but are not identified with reliability, they are only suggestive. 
BB Doradus BB Dor was observed with FUSE in a high state. We fit here four different models as shown in Table 6: a WD, a disk, a WD+Disk at low inclination, and a WD+Disk at high inclination. The high inclination disk contributes much less flux than the WD, while the low inclination disk contributes much more flux than the WD. The sharp emission lines, all colored in blue, are most likely due to the reflection of day and/or sun light in the telescope and therefore marked with a $\mathrm{C}$ in Table 4 . There are lines that appear clearly in the model but cannot be identified with reliability in the observed spectrum; these lines are marked on the figure but appear with a D in Table 4 (e.g. S IV 1006 \& 1100). The S IV (1063 $\&$ 1073) and Si III (1110) lines are much deeper in the observed spectrum than in the model, possibly a sign that there is additional absorption from material above the disk. The oxygen doublet is also in absorption and is not modeled. All the details of the spectral modeling, data analysis and processing of the data are given in Godon et al. (2008a).

BV Centauri The FUSE Spectrum of the U Gem dwarf nova BV Centauri was taken in quiescence. The spectrum is very noisy especially at the shorter wavelengths. The increase of flux at at $920 \AA$ is probably due to a background subtraction problem. We identify ISM Hydrogen molecular absorption features as well as some ISM iron lines. The sharp emission lines are due to terrestrial airglow. The broad O vi doublet and C III (977 $\AA$ ) are from the source. The S IV (1073), Si III (1113), Si IV (1123 \& 1128) and C III (1175) absorption spectra features are all identified. All the other absorption features marked on the figure are suggestive and are not detected. The modeling takes into account the larger mass recently found of $1.24 M_{\odot}$ by Watson et al. (2007a), this does not change the temperature of the WD appreciably, but it reduces the distance to about 200 pc. The modeling includes the effect of the ISM and the theoretical spectrum that is compared to the observed one consists in the spectral regions colored in red (in the second online figure). Details on the processing and spectral modeling of the FUSE spectrum are given in Sion et al. (2007).

CH Ursa Majoris The FUSE Spectrum of the U Gem Dwarf Nova CH Ursa Majoris was taken in quiescence. The spectrum is very noisy especially in the shorter wavelengths. Due to the hydrogen cut-off one expects the flux to drop significantly for wavelengths $<915 \AA$, instead the flux keeps on increasing, a sure sign of a noisy detector edge as well as a possible background subtraction problem. We identify ISM H molecular absorption features as well as ISM metal lines, mainly of Fe II. The spectrum is also affected by terrestrial airglow (sharp emission lines) and the S vi (944) feature, if real, is also probably due to day light reflected in the telescope. There are some broad emission features that are associated with the source: we identify the O vi doublet and C III (977 \& $1175 \AA$ ). All the other lines marked in the figure are not detected. In the modeling we add an ISM model to mimic the absorption 
due to the Hydrogen molecular absorption features. Details on the processing and spectral modeling of the FUSE spectrum are given in Sion et al. (2007).

DQ Herculis The FUSE spectrum of the IP DQ Her has strong and broad emission lines. These include Niv, S vi, CiII, HeII, and O vi lines. The continuum of the flux is very low and shows some weaker emission lines from PIV, PV, S IV and Si III. The emission lines originate near the poles where matter accretes through magnetic lines.

DT Apodis DT Aps was observed twice with FUSE, but only the second FUSE spectrum shows a continuum. This low $\mathrm{S} / \mathrm{N}$ spectrum is heavily affected by air glow, especially at the shorter wavelengths. The deep absorption feature (1110-1130 $\AA$ ) is probably due to an incorrect background subtraction (as is probably the C III feature). One cannot identify with reliability any spectral line from the source. The system parameters are unknown and, therefore, one cannot model the spectrum as there is a degeneracy in the modeling. Details on the processing of the observed spectrum are given in Godon et al. (2009a).

EK Trianguli Australis The FUSE spectrum of the DN EK TrA in quiescence is modeled with a WD. The effect of the Hydrogen quasimolecular opacity has to be included to agree with the flux around 1060-1080 $\AA$. The theoretical spectrum without the inclusion of the $\mathrm{H}$ quasimolecular absorption is shown with a dashed line in that region (1060-108 $\AA$ ). All the sharp emission lines (including the nitrogen lines) are due to airglow. The broad lines of C III (977 \& 1175) and O VI are from the source. In the longer wavelengths of FUSE the theoretical and observed spectra match each other in some regions but not everywhere. For example the S IV has an observed flux lower than modeled at $1123 \AA$, while at the bottom of the broad absorption feature at $1128 \AA$ there is a small sharp emission feature most probably due to the low $\mathrm{S} / \mathrm{N}$. These lines are identified by their location and the by the overall shape of the continuum in their immediate neighborhood. The spectrum is a combination of two FUSE spectra, and has been dereddened assuming the published value $\mathrm{E}(\mathrm{B}-\mathrm{V})=0.03$. Details of the modeling are given in Godon et al. (2008b).

EM Cygni The FUSE data of EM Cyg were obtained during a relatively low state of activity. The spectrum (see Figure 5) consists of a FUSE exposure taken at orbital phase 0.15 when the flux was maximum. The other FUSE exposures have a lower flux. The FUSE spectrum is strongly affected by ISM H molecular absorption which is included in the modeling. The NIV (923) and S VI (933) lines are not identified due to the low S/N, and S Vi (944) could possibly be in absorption. The C III (977 \& 1175) and O vi lines are seen 
each in absorption on top of a broader emission feature. The only lines that we identified in absorption (and are also deeper than in the model) are S IV (1073), Si III $(\sim 1110)$ and Si IV (1123 \& 1128). The other lines that are usually observed are marked on the figure but are not identified. The model spectrum consists of a WD+Disk. The WD component contributes $93 \%$ of the flux, the disk contributes the remaining $7 \%$. Some absorption lines cannot be accounted for and are possibly due to veiling of the WD from the L1 stream material flowing over the disk to smaller radius at different orbital phases. Maximum veiling is around orbital phase 0.6-0.9. All the details of the processing of the FUSE spectra and their analysis is given in Godon et al. (2009b).

ES Draconis ES Dra was observed in an intermediate state and it is not clear whether the main component is a WD or a disk, as the inclination and WD mass of the system are not known. Because of that, the FUSE spectrum of ES Dra is modeled first with a single WD, and then with a DISK alone. We do not consider any WD+disk model, as there is a degeneracy of the solution (there are many WD+disk models that can fit this spectrum covering a large area in the parameter space). Details on the processing of the observed spectrum and the spectral analysis are given in Godon et al. (2009a). The spectrum exhibits some sharp emission lines due to air glow as well as ISM absorption lines (mainly molecular H, but also some metals). There could be some broad emission near $977 \AA(\mathrm{C}$ III ?) as well as near $990 \AA$. The C III (1175) feature seem to have blue shifted absorption component with a red shifted emission component. We identify with some reliability absorption lines/features from C II (1010),Si IV (1066), Si III ( 1110\& 1143), Si IV (1123-1128), as these lines are located at the expected wavelengths and agree grossly with the model. The SIV $(1063,1073)$ lines are contaminated with ISM absorption. Some of the lines that are marked on the figure are present in the theoretical spectrum but are not detected in the observed spectrum (e.g. S IV $\sim 1006$ \& 1100).

EX Hydrae The IP EX Hya was observed with FUSE during both a lower and higher state, as it changed with the orbital phase. The spectrum here was obtained in the low state in which the WD is possibly revealed and contributes a significant fraction of the FUV flux. The spectrum has relativelty good $\mathrm{S} / \mathrm{N}$. There is a sharp hydrogen Lyman $\beta$ emission line due to air glow. C III (977 \& 1175) and the O vi doublet are characterized by a shallow absorption on top of a braoder emission. The oxygen doublet also exhibits a narrow emission slightly red shifted. The atomic hydrogen and oxygen narrow absorption lines are from the ISM, and so are the CII (1036 \& 1037) and nitrogen lines (though some of the nitrogen lines could be contaminated from the terrestrial atmosphere). The system exhibits narrow absorption lines (broader than the ISM lines) of highly ionized species such as S VI (933, 
944), P IV, P v, O vi, S IV, Si IV as well as lines of Si II, Si III, S III, S II, C II, C III and He II. This indicates that there are several absorbing layers with different temperatures.

EY Cygni This low S/N FUSE spectrum of EY Cyg was obtained in quiescence and is modeled with a WD spectrum. The increase of flux in the short wavelengths $(<950 \AA)$ is an artifact in one of the FUSE channels due to inaccurate background subtraction, the N IV (923) lines have been marked but are not detected and do not match any observed feature. All the sharp emission lines of $\mathrm{H}, \mathrm{O}, \mathrm{He}$ ) are due to geo- and helio-coronal emission (light reflected in the telescope). There could be some broad emission from O vi (1032) and, though less likely, from O vi (1038). We have marked some of the ISM absorption lines where they match some absorption features in the observed spectrum such as some hydrogen molecular lines and CII (1036). With some low (but non-zero) reliability we identify some absorption lines matching the theoretical spectrum as follows: S IV (1073), Si IV (1128) and Si III ( 1142-1145). All the other lines that are marked on the figure are not identified and are there mostly for comparison and identification with the theoretical spectrum. The FUSE spectrum of this system was processed and modeled in Godon et al. (2008b). This object exhibits the $\mathrm{N} / \mathrm{C}$ composition anomaly indicative of CNO processing.

FO Persei FO Per was observed with FUSE in a high state and was modeled with an accretion disk and a heated WD, assuming both $\mathrm{E}(\mathrm{B}-\mathrm{V})=0.0$ and $\mathrm{E}(\mathrm{B}-\mathrm{V})=0.3$. Details on the processing of the observed spectrum and the spectral analysis are given in Godon et al. (2009a). This FUSE spectrum is rather noisy and has been binned to a lower resolution for slightly increase the $\mathrm{S} / \mathrm{N}$. All the sharp emission lines are most likely day light contamination (all the H i lines, the S VI, O vi, C III and He I lines). We identify only the Si IV (1066), Si IV (1123 \& 1128) and C III lines with the source. All the other lines that are marked are either contaminated with ISM absorption (mostly molecular $\mathrm{H}$ ), terrestrial in origin (e.g. NI), or are of the order of the noise. All the features that are not from the source have been marked in blue in the figure and were masked before the modeling (spectral fit).

HP Normae HP Nor was observed in a relatively high state and was modeled with an accretion disk. The FUSE spectrum was dereddened assuming $\mathrm{E}(\mathrm{B}-\mathrm{V})=0.2$. Details on the processing of the observed spectrum and the spectral analysis are given in Godon et al. (2009a). The FUSE spectrum has a very low S/N and is contaminated with sharp emission lines (geo- and helio-coronal in origin). The ISM molecular hydrogen absorption features are slicing the spectrum at regular intervals. The only feature that can can be associated with the source and that somehow matches the model (withint the $\mathrm{S} / \mathrm{N}$ range) is the $\mathrm{C}$ III (1175) 
multiplet. The ISM absorption features and the day light contamination have been marked in blue in the figure and have been masked before the fitting.

IX Velorum In this high S/N FUSE spectrum of IX Vel, the sharp absorption lines are all observed at their theoretical rest wavelengths, while the broad absorption lines are blue shifted by about $2 \AA$ and exhibit a sharp steep red wing and a much broader blue wing. All the broad absorption lines are associated with the source and have been marked on the figure at the rest wavelength such that the blue shift is evident. All the sharp absorption lines are from the ISM, except possibly for the nitrogen lines (terrestrial). Processing of the FUSE spectrum is given in Linnell et al. (2007) together with a spectral modeling of the FUSE spectrum combined with a STIS and IUE spectra.

MU Camelopardalis The FUSE spectrum of the IP MU Camelopardalis is of a very low quality. All the sharp emission lines are airglow contamination, possibly including S vI, $\mathrm{C}$ III and O vi from sunlight reflected on the $\mathrm{SiC}$ channels. Though the $\mathrm{S} / \mathrm{N}$ is low, we clearly identify ISM molecular hydrogen, as well as absorption lines from C II. No S IV lines are detected. Except for the broad Ovi doublet emission lines, no other spectral feature can be associated with the source. The continuum is rather flat and could indicate little contribution from the accreting WD.

MV Lyrae This FUSE spectrum of the Nova-like (VY) MV Lyr was taken during a low state. The spectrum (see Figure 3) reveals the accreting WD, which is heated due to continuous accretion as typical for NL VY systems. There is no discernable extinction toward MV Lyr. The spectrum consists of four exposures (FUSE orbits). The WD model (solid white line) has a mass of $0.73 M_{\odot}$, a temperature of $45,000 \mathrm{~K}$, a rotational velocity of $200 \mathrm{~km} \mathrm{~s}^{-1}$ and solar composition. To fit the flux below $930 \AA$ one has to decrease the temperature to 44,000 K. The slight decrease of flux (between 915 and $930 \AA$ ) is possibly due to the sharp H I absorption lines. In order to fit the right wing of the Ly $\beta$ profile one has to increase the temperature to $47,000 \mathrm{~K}$. The right wing might be affected by some O VI emission. All the sharp emission lines (H I, C III, and more) are most probably from air glow. We identify the following absorption lines which fit the WD photosphere model: S IV (1006), C II (1010), S IV (1063, 1073), Si IV (1066), He II (992, 1085), Si III (1110), P V (1118), Si IV (1123, 1128) and CIII (1175). All the other lines are either not identified or they are sharp ISM absorption lines (e.g. Si II, C II, Ar I, Fe II). For solar composition, the WD has to have a rotation rate of at least $200 \mathrm{~km} \mathrm{~s}^{-1}$ and possibly as fast as $250 \mathrm{~km} \mathrm{~s} s^{-1}$ in order to match the profile of the absorption lines. If one decreases the abundances, the 
rotational velocity needed to match the absorption lines decreases as well. For $\mathrm{Z}=0.2$ the (projected) rotational velocity needed to match the lines is $150 \mathrm{~km} \mathrm{~s}$. For an inclination of $12^{\circ}$, a projected rotational velocity of $200 \mathrm{~km} \mathrm{~s}^{-1}$ corresponds to about $1 / 3$ of the Keplerian velocity at one stellar radius. For a velocity of $150 \mathrm{~km} \mathrm{~s}^{-1}$, it corresponds to about $1 / 4-1 / 5$ of the Keplerian velocity. Note that if one adopts $i=7^{\circ}$ as in Linnell et al. (2005) then $250 \mathrm{~km} \mathrm{~s}^{-1}$ corresponds to $2 / 3$ of the Keplerian velocity. It is more likely that the inclination is $12^{\circ}$ and the projected rotational velocity $150 \mathrm{~km} \mathrm{~s}^{-1}$, and that the WD rotates at a fraction of about $1 / 4$ to $1 / 5$ of the breakup velocity.

NSV10934 NSV 10934 was observed with FUSE twice consecutively, and we combined the two individual spectra. At the time of the observations NSV 10934 was in a low state about a month after an outburst. The low $\mathrm{S} / \mathrm{N}$ (especially in the lower wavelengths) is due to the fact that the data from the $2 \mathrm{bSiC}, 2 \mathrm{bLiF}$ and $1 \mathrm{aSiC}$ channels were unusable and a large portion of the $1 \mathrm{bLiF}$ channel was lost to the infamous worm. Because of this there is a gap around $1085 \AA$ and the spectrum is not modeled. Details on the processing of the observed spectrum can be found in Godon et al. (2009a). The only spectral feature that we identify and which belong to the source are the braod emission from C III (977 \& 1175) and the O vi doublet. The other spectral features that are identified are the ISM molecular hydrogen absorption lines. All the other other spectral lines marked on the figure are only suggestive.

P831 57 The FUSE spectrum of the DA WD + dMe binary P831 57 was taken as part of a NL FUSE survey as the system was classified as a NL. From the present analysis it was found that the system does not have a disk and the WD is accreting from the wind of the secondary. The system also appears in the Downes catalog as NL called Ret 1, and it is also known under the name WD 0334-6400. The spectrum together with the model is shown in Figure 2 and details on the analysis can be found in Barrett et al. (2012). On the figure the following lines are marked but are NOT identified: N IV (923), S vi (933, 944), O vi doublet. All the sharp emissin lines are either helio coronal or air glow. The spectral feature around $1152 \AA$ is a well known fixed pattern noise (FPN) in FUSE, a smaller FPN feature is also detected around $1072 \AA$. Since the WD has an extremely low rotation, its absorption lines are very sharp, similar to ISM lines. However, only the WD lines match the theoretical spectrum: C III (977, 1175), N III + He II (990), Si III ( 995), S IV (1006), C II (1010), C II (1036), S IV (1063, 1073), Si IV (1066), N II + He II ( 1085), Si III (1008, 1010, 1013), Si IV $(1023,1028)$ and N I (1134). Some of these lines are deeper in the observed spectrum than in the model, possibly an indication that they are superposed to ISM lines (e.g. C II 1036, N I 1134). All the other sharp lines are from the ISM (N I 954, Ar I, etc..). 
RU Pegasi This FUSE spectrum is rather noisy and is heavily affected by ISM absorption lines from atomic and molecular hydrogen, as well as from metals (Ar I, Fe II, S I, Ni). The C III (977 \& 1175) and oxygen doublet lines are seen in broad emission with a narrower absorption feature superposed to it. We identify absorption lines from N IV (923), S vi (933, 944), oxygen doublet, and C III (1175) which are all slightly red shifted (by about $1 \AA$ ), an indication that they possibly do not belong to the WD photosphere per se. We do, however, identify some absorption lines from the WD that are not shifted: Si IV (1066), O IV (1068) and S IV (1073). The O IV line do appear in very hot WDs and is a sign that the WD in RU Peg is indeed relatively hot and is usually not observed in accreting WDs in CVs. The FUSE spectrum of the DN RU Peg in quiescence is modeled with a WD spectrum. The very high temperature of the model is needed to fit the flux level of a massive (and therefore compact) WD (i.e. to fit the distance) and to account and fit properly the S IV (1073), Si IV (1066), and O VI (1068) absorption lines. The FUSE spectrum of this system was processed and modeled in Godon et al. (2008b).

RW Sextantis The spectrum of RW Sex consists of 25 FUSE exposures (orbits) combined together. From exposure to exposure the continuum remains fairly constant and only the bottom of the broad lines shows some variation. The spectrum shows broad absorption lines from high order ionization species (all blueshifted here by about $3 \AA$ ): N IV, S vi, C III, N III, O vi, S IV, P IV \& PV. The spectrum is also rich in ISM lines: molecular hydrogen, oxygen and some metal lines such as CII, Ar I, Fe II and many P II lines. The broad absorption lines can be seen better when considering the difference between exposures as a function of the orbital phase (due to the inclination of the system). Since only the broad absorption lines vary with the phase, a difference graph clearly shows the broad lines. In the difference graph the absorption lines appear as broad emission and are blue shifted by about $1.5 \AA$. The FUSE spectrum of RW Sex combined with existing HST and IUE spectra was analyzed in Linnell et al. (2010).

SS Aurigae The FUSE spectrum of the DN SS Aur was taken in quiescence and it is modeled with a WD spectrum assuming $\mathrm{E}(\mathrm{B}-\mathrm{V})=0.08$. We identify some broad emission features from C III (977) and the oxygen doublet, and identify (with matching the synthetic spectrum) absorption lines from C II (1010, usually seen in lower temperature WDs),Si III (1113, and 1145$)$, and Si IV (1123 \& 1128). The C III (1175) line is not detected at all and the continuum there appear to be fairly flat. In spite of the low $\mathrm{S} / \mathrm{N}$ we do identify many ISM molecular hydrogen and some metal absorption lines. The FUSE spectrum of this system was processed and modeled in Godon et al. (2008b). 
SS Cygni The spectrum consists of eight individual FUSE exposures (orbits) combined together. The system was in a relatively low state at the time of the observations. The FUSE spectrum is characterized by a relatively low flux with broad emission lines (Hydrogen Lyman series, C III, N III and Ovi doublet). The spectrum has a "forest" of ISM lines: molecular hydrogen (marked in the top of each panel in the figure), atomic hydrogen (marked below each panel), oxygen (O I) and few metal lines (marked in the lower part of the panels). The spectrum has been dereddened assuming $\mathrm{E}(\mathrm{B}-\mathrm{V})=0.04$. The main spectral features that belong to the source are the braod emission lines from C III (977 and 1175) and the oxygen doublet. The absorption lines that are usually seen in WD photosphere (e.g. from S and $\mathrm{Si}$ ) are not detected at all and the continuum appear to be rather featureless. A composite WD+Disk model has been fit to this spectrum. The disk contributes only $12 \%$ of the flux while the WD contributes the remaining $88 \%$ of the flux. The increase in flux in the shorter wavelengths of FUSE could be due to broad and strong emission lines of N IV, S VI, C III and the higher order of the Hydrogen Lyman series merging together to form a continuum, but it could also be due to the tail of a hotter source. The distance obtained from this fit is $173 \mathrm{pc}$, in excellent agreement with its trigonometric parallax distance. The complete analysis is given in Sion et al. (2010).

TX Columbae The FUSE spectrum of the intermediate polar TX Col is rather poor, in many respects, resembling the spectrum of MU Cam, both in its quality and in its features. The ISM lines have been marked without labeling. There seems to be some NIV, N III, He II and O vi emission, as well as possibly some S IV (1073 $\AA$ ) emission, but little C III. Additional lines have been marked but are not detected with confidence.

UU Aquilae The FUSE spectrum of the U Gem-type dwarf nova UU Aquilae was taken during quiescence. The spectrum is very noisy at the shorter wavelengths. The sharp emission lines are geo- and helio-coronal in origin. We identify ISM hydrogen molecular absorption features polluting the spectrum over almost the entire FUSE range. We note the absence of the CIII 1175 absorption line. The spectrum was modeled using a single WD component. Details on the processing and spectral modeling of the FUSE spectrum of UU Aql are given in Sion et al. (2007). In a first model we assumed a 1 solar mass WD $(\log (g)=8.6)$ with solar composition except for $\mathrm{C}=0.001$ solar. We obtain a temperature of 29,000 $\mathrm{K}$ and a distance of $380 \mathrm{pc}$. When we assume a canonical mass of 0.6 solar mass, we obtain a temperature of $26,000 \mathrm{~K}$ and a distance of $450 \mathrm{pc}$ (this model is very similar to the 1 solar mass model). The apparent discrepancy between the model and the observed spectrum shortward of $1040 \AA$ is due mainly to ISM Hydrogen molecular absorption. The Si III and S IV lines between $1100 \AA$ and $1150 \AA$ are relatively well modeled. 
V3885 Sgr The FUSE spectrum of the nova-like V3885 Sgr is shown in Figure 6 with a $\mathrm{WD}+$ disk model fit including the contribution from a hot boundary layer. The $0.7 M_{\odot} \mathrm{WD}$ model has a temperature of $60,000 \mathrm{~K}$, a projected rotational velocity of $200 \mathrm{~km} \mathrm{~s} \mathrm{~s}^{-1}$, and solar abundances. The disk model has $\dot{M}=3 \times 10^{-9} M_{\odot} /$ yr with an inclination of $60^{\circ}$ and includes two inner (boundary layer) rings with $\mathrm{T}=175,000 \mathrm{~K}$. The WD (dotted line) contributes only $22 \%$ of the flux and the disk+BL (dashed line) contributes $78 \%$. The sharp absorption lines are from the ISM. All the broad absorption lines originate from a corona above the disk and/or boundary layer and are blue shifted by $\sim 1-1.5 \AA$. The lines are all marked at their rest wavelengths to emphasize the shift. All the absorption lines are colored in blue and only the continuum (in red) is modeled.

V405 Aurigae The FUSE spectrum has broad emission lines from highly ionized species CiII, O vi, Siv, N III together with very sharp emission lines possibly mostly from airglow contamination (Hydrogen Lyman series, O I series, S vi). There are many ISM molecular hydrogen abosprtion lines. Ar I, N I, and Si II lines are also from the ISM. The relatively low $\mathrm{S} / \mathrm{N}$ of the continuum prevents us from identifying more features.

V471 Tauri V471 Tau was observed under the FUSE program M112 (Periodic Channel Coalignment) together with other bright UV sources to check the alignment of the FUSE channels. During the observation the FUSE telescope was performing X and Y scans in the field of view of the target and therefore the FUV flux of V471 Tau was collected only a fraction of the time. The present FUSE spectrum was obtained by integrating only during the time V471 Tau was on target with a constant FUV flux. In the first and second exposures we selected 250s each of good exposure time, in the $3 \mathrm{rd}-300 \mathrm{~s}$, in the 4 th $-800 \mathrm{~s}$, and in the 5 th and last exposure 500s, totaling 2145s of good exposure time. The FUSE spectrum of V471 Tau (see Figure 1) is mainly that of a WD atmosphere with Hydrogen, rotating slowly. There is little absorption except for the orders of the Lyman series. The Si and C lines were fit using very low abundances (see Table 6) and with a rotational velocity of $250 \mathrm{~km} \mathrm{~s}$, which is about four times larger than expected. The broadening of the lines could possibly be due to an unresolved Zeeman splitting as suggested by Sion et al. (2012) who carried out an analysis of the HST/STIS spectra of V471 Tau.

V794 Aquilae The FUSE spectrum of the NL V794 Aql in a high state is shown with a disk spectrum. The FUSE spectrum has been dereddened assuming $\mathrm{E}(\mathrm{B}-\mathrm{V})=0.20$ estimated from the $2200 \AA$ feature in the IUE spectra. Details of the processing of the FUSE spectrum and its spectral modeling are given in Godon et al. (2007). The spectrum exhibits many sharp 
emission lines possibly all from terrestrial/solar contamination. The spectrum is basically sliced by ISM molecular hydrogen absorption lines and also includes some ISM metal lines such as Ar I, Si II, Fe II, P II, N I and S I. There is no obvious broad emission lines and only a comparison with the disk model shows reveals that there could be some extra flux in the region of the oxygen doublet and $\mathrm{C}$ III.

VW Hydri The FUSE spectrum of the DN VW Hyi is shown in quiescence with a single WD model. We ignore a possible second component (see the analysis of Long et al. (2007, 2009)). The effect of the the Hydrogen quasimolecular opacity is included in the modeling and they way it affects the spectrum is indicated with the arrows (the dotted/dashed line shows a model without its inclusion). The FUSE spectrum has been dereddened assuming the published value $\mathrm{E}(\mathrm{B}-\mathrm{V})=0.01$. There are many more FUSE exposures available in the MAST archive, however, the FUSE spectrum in the catalog was processed and modeled in Godon et al. (2008b). The additional FUSE spectra of VW Hyi were analyzed in Long et al. (2007, 2009). Towards the shorter wavelengths as well as in the Lyman $\beta$ region the flux does not go to zero. It could be due to a second emitting component (emitting a continuum flux). This second component is observed only during the early stages after outburst.

WW Ceti The FUSE spectrum of the DN WW Cet in quiescence is shown with a single WD model. The effect of the Hydrogen quasimolecular opacity is included in the modeling. Towards the very short wavelengths one does not expect much flux due to the hydrogen cut-off. The fact that we do detect flux there is a sign of a noisy spectrum due to wrong background substraction and/or to a noisy detector edge. Since the flux in the bottom of the Lyman $\beta$ does not go to zero (at least on the left side not affected by oxygen emission), a second component could also be present here contributing flux at wavelengths $<950 \AA$. We ignore here a possible second component (the second component is modeled in Godon et al. (2006)). We do not identify any feature due to N IV (923), S vi (933, 944), S IV (1006), C II (1010), and Si III (1158). All the other lines marked on the figure are identified.

YY Draconis The FUSE exposures of the IP YY Dra was heavily affected by airglow and the spectrum presented here has been processed using the "NIGHT" option in CalFUSE. The resulting spectrum has basically no airglow but the $\mathrm{S} / \mathrm{N}$ is not very high. The spectrum is characterized by a rather flat continuum with three broad emission features from CIII

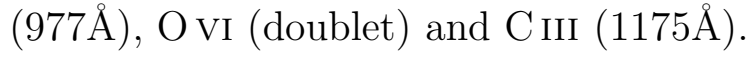




\section{REFERENCES}

Abgrall, H., Roueff, E., \& Drira, I. 2000, A\&AS, 141, 297.

Allen, D.A. 1978, MNRAS, 184, 601

Allen, D.A. 1980, MNRAS, 192, 521

Allen, D.A. \& Glass, I.S. 1974, MNRAS, 167, 337

Andronov, I.L. 1991, Commission 27 of the IAU, Information Bulletin on Variable Stars, p.3645

Andronov, I.L., \& Chinarova, L.L. 1995 ASSL 205, 150

Andronov, I.L., \& Chinarova, L.L. 1996, OAP 9, 9

Andronov, I.L. et al. 2003, ASP Conference Series, Vol. 292, Interplay of Periodic, Cyclic and Stochastic Variability in Selected Areas of the H-R Diagram, ed. C. Sterken, p.313

Barrett, P.E., Dupuis, J., Schlegel, E.M., Heathcote, B.D., Godon, P., Sion, E.M. 2012, ApJ, submitted

Barstow, M.A., Schmitt, J.H.M.M., Clemens, J.C., Pye, J.P., Denby, M., Harris, A.W., Pankiewicz, G.S. 1992, MNRAS, 255, 369

Barstow, M.A., Holberg, J.B., Cruise, A.M., Penny, A.J., 1997, MNRAS, 290, 505

Bateson, F.M., 1979, Ro.Astron.Soc. of New Zealand - Publ. Variable Star Sect., 7, 20

Bateson, F.M., \& McIntosh, R. 1990, Ro.Astron.Soc. of New Zealand - Publ. of Variables Star Section, 17, 76

Bayley, J.A. 1981, MNRAS, 197, 31

Becker, R.H., Chanan, G.A., Wilson, A.S., Pravdo, S.H. 1982, MNRAS, 201, 265

Belczyński, K., Mikolajewska, J., Brandi, E., Garcia, L.G., Ferrer, O.E., Pereira, C.B. 2000, New AR, 44, 81

Belloni, T. et al. 1991, A\&A, 246, L44

Bengtsson, H., 1975, Astronomisk Tidsskrift 8, 74

Beuermann, K. \& Pakull, M.W. 1984, A\&A, 136, 250 
Beuermann, K., \& Thomas, H.-C. 1990, A\&A, 230, 326

Beuermann, K., Stasiewski, U., \& Schwope, A.D. 1992, A\&A, 256, 433

Beyer, M., 1967, Astronomische Nachrichten, 290, 29

Bitner, M.A., Robinson, E.L., \& Behr, B.B. 2007, ApJ, 662, 564

Bochkarev, N.G., \& Sitnik, T.G. 1985, Ap\&SS, 108, 237

Bois, B., Mochnacki, S.W., Lanning, H.H. 1988, AJ, 96, 157

Bolick, U., Beuermann, K., Bruch, A., Lenzen, R. 1987, Ap\&SS, 130, 175

Bond, H.E., Mullan, D., O’Brien, S., \& Sion, E.M. 2001, ApJ, 560, 919

Brady, R.A., Herczeg, T.G. 1977, PASP, 89, 71

Bruch, A. 1989, A\&AS, 78, 145

Bruch, A., \& Engel, A. 1994, A\&AS, 104, 79

Bruch, A., Fischer, F.-J., Wilmsen, U. 1987, A\&A, 70, 481

Burbidge, E.M., Burgidge, G.R. 1953, ApJ, 118, 349

Cannizzo, J.K., \& Mattei, J.A. 1992, ApJ, 401, 642

Cannizzo, J.K. 1993, ApJ, 419, 318

Cannon, A.J. 1933, Harvard Obs. Bull., 891, 9

Cester, B. \& Pucillo, M. 1976, A\&A, 46, 197

Cheng, A., O’Donoghue, D., Stobie, R.S., Kilkenny, D., Warner, B., 2001, MNRAS, 325, 89

Chiappetti, L., Maraschi, L., Treves, A., Tanzi, E.G. 1982, ApJ, 258, 236

Clemens, J.C. et al. 1992, ApJ, 391, 773

Costero, R., Echevarria, J., \& Pineda, L. 1998, AAS, 192, 8206

Costero, R., Echevarria, J., Michel, R., Zharikov, S. 2004, AAS, 205, 1906

Csizmadia, Sz., Nagy, Sz., Borkovits, T., Hegedüs, T., Biró, I.B., Kiss, Z.T. 2008, Astronomische Nachrichten, 329, 39 
Davis, J.F., Mattei, J.A. 1981, AAVSO Journal, 10, 28

Dixon, W.V., et al. 2007, PASP, 119, 527

Downes, R.A., Webbink, R.F., Shara, M.M., Ritter, H., Kolb, U., Dürbeck, H.W. 2001, PASP, 113, 764

Dupuis, J., Vennes, S., Chayer, P., Cully, S., \& Rodriguez-Bell, T. 1997, in White Dwarfs, Eds J. Isern, M. Hernanz \& E. Garcia-Berro (Dordrecht: Kluwer), 375

Eggen, O.J., \& Niemela, V.S. 1984, AJ, 89, 389

Fekel, F.C., Hinkle, K.H., Joyce, R.R. 2003, ASPC 303, 113

Fekel, F.C., Hinkle, K.H., Joyce, R.R., Wood, P.R., 2010, AJ, 139, 1315

Friend, M.T., Martin, J.S., Connon-Smith, R., Jones, D.H.P. 1990, MNRAS, 246, 654

Froning, C.S., Long, K.S., Gänsicke, B.T., Szkody, P. ApJS, 199, 7

Galis, R., Hric, L., Friedjung, M., Petrik, K. 1999, A\&A, 348, 533

Gänsicke, B.T., \& Beuermann, K. 1996, A\&A, 309, L47

Gänsicke, B.T., Beuermann, K., \& Thomas, H.-C. 1997, MNRAS, 289, 388

Gänsicke, B.T., Long, K.S., Barstow, M.A., Hubeny, I. 2006, ApJ, 639, 1039

Gänsicke, B.T., Szkody, P., de Martino, D., Beuermann, K., Long, K.S., Sion, E.M., Knigge, C., Marsh, T., \& Hubeny, I. 2003, ApJ, 594, 443

Gänsicke, B.T., Szkody, P., Sion, E.M., Hoard, D.W., Howell, S., Cheng, F.H., \& Hubeny, I. 2001, ApJ, 374, 656

Garrison, R.F., Hiltner, W.A., \& Schild, R.E. 1982, IAU Circ. 3730, 2

Garrison, R.F., Schild, R.E., Hiltner, W.A., Krzeminski, W. 1984, ApJ, 276, L13

Gessner, H. 1978, Mitt. Veraenderl. Sterne, 8, 66

Gilliland, R. L. 1982, ApJ, 263, 302

Godon, P., Seward, L., Sion, E.M., Szkody, P., 2006, AJ, 131, 2634

Godon, P. \& Sion, E.M. 2005, MNRAS, 361, 809 
Godon, P., \& Sion, E.M. 2011, PASP, 2011, 123, 903

Godon, P., Sion, E.M., Barrett, P.E., Szkody, P. 2007, ApJ, 656, 1092

Godon, P., Sion, E.M., Barrett, P.E., Szkody, P., Schlegel, E.M. 2008a, ApJ, 687, 532

Godon, P., Sion, E.M., Barrett, P.E., Hubeny, I., Linnell, A.P., Szkody, P. 2008b, ApJ, 679, 1447

Godon, P., Sion, E.M., Barrett, P.E., Szkody, P., 2009a, ApJ, 701, 1091

Godon, P., Sion, E.M., Barrett, P.E., Linnell, A.P., 2009b, ApJ, 699, 1229

Godon, P., Sion, E.M., Cheng, F.H., Szkody, P., Long, K.S., \& Froning, C.S. 2004, ApJ, 612,429

González-Riestra, R., Viotti, R., Iijima, T., Greiner, J. 1999, A\&A, 347, 478

Green, R.F., Schmidt, M., \& Liebert, J. 1986, ApJS, 61, 305

Greenstein, J.L., \& Oke, J.B. 1982, ApJ, 258, 209

Greiner, J., Bickert, K., Luthardt, R., Viotti, R., Altamore, A., González-Riestra, R., Stencel, R.E. 1997, A\&A, 322, 576

Guinan, E.F., \& Sion, E.M. 1982, ApJ, 258, 217

Guinan, E.F., \& Sion, E.M. 1984, AJ, 89, 1252

Hacke, G. 1987, Astronomicheskii Tsirkulyar, 1491, 6

Hamada, T., \& Salpeter, E.E. 1961, ApJ, 134, 683;

Hamilton, R.T., Urban, J.A., Sion, E.M., Riedel, A.R., Voyer, E.N., Marcy, J.T., Lakatos, S.L. 2007, ApJ, 667, 1139

Harrison, T.E., McNamara, B.J., Szkody, P., McArthur, B.E., Benedict, G.F., Klemola, A.R., Gilliland, R.L. 1999, ApJ, 515, L93

Harrison, T.E., McNamara, B.J., Szkody, P., \& Gililand, R. 2000, AJ, 120, 2649

Harrison, T.E., Johnson, J.J., McArthur, B.E., Benedict, G.F., Szkody, P., Howell, S.B., Gelino, D.M. 2004, AJ, 127460

Harrison, T.E., Osborne, H.L., Howell, S.B. 2005, AJ, 129, 2400 
Hartley, L.E., Drew, J.E., Long, K.S., Knigge, C., Proga, D. 2002, MNRAS, 332, 127

Hartley, L.E., Murray, J.R., Drew, J.E., \& Long, K.S. 2005, MNRAS, 363, 285

Hassal, B.J.M. 1985, MNRAS, 216, 335

Haug, K. 1987, Ap\&SS, 130, 91

Haug, K. 1988, MNRAS, 235, 1385

Haug, K., \& Drechsel, H., 1985a, Mitteilungen der Astronomischen Gesellschaft, 63, 193

Haug, K., \& Drechsel, H. 1985b, A\&A, 151, 157

Hawkins, M.R., Smith, R., \& Jones, D.H.P. 1990, in Accretion-Powered Compact Binaries, ed. C. Mauche (Cambridge: Cambridge Univ. Press), 113

Herczeg, T.J. 1987, Ap\&SS, 130, 39

Hoard, D.W., Linnell, A.P., Szkody, P., Fried, R.E., Sion, E.M., Hubeny, I., Wolfe, M.A., 2004, ApJ, 604, 346

Hoard, D.W., Szkody, P., Froning, C.S., Long, K.S., \& Knigge, C. 2003, AJ, 126, 2473

Hoffmeister, C. 1928, Astron.Nach., 234, 33

Holm, A.V. 1988, in ESA, A Decade of UVAstronomy with the IUE Satellite, ed. E.J.Rolf (ESA SP-281; Paris: ESA), 229

Holm, A.V., \& Polidan, R.S. 1988, in ESA, A Decade of UVAstronomy with the IUE Satellite, ed. E.J.Rolf (ESA SP-281; Paris: ESA), 179

Honeycutt, R.K., \& Schlegel, E.M. 1985, PASP, 97, 1189

Honeycutt, R.K., Cannizzo, J.K., \& Robertson, J.W. 1994, ApJ, 425, 835

Honeycutt, R.K., \& Robertson, J.W. 1998, ApJ, 116, 1961

Howarth, I.D. 1976, Mitt. Veraenderl. Sterne, 7, 147

Howell, S.B., \& Szkody P. 1990, ApJ, 356, 623

Huang, M., Sion, E.M., Hubeny, I., Cheng, F., \& Szkody, P. 1996a, ApJ, 458, 355

Huang, M., Sion, E.M., Hubeny, I., Cheng, F.-H., \& Szkody, P. 1996b, AJ, 111, 2386 
Hubeny, I. 1988, Comput. Phys. Commun., 52, 103;

Hubeny, I.,\& Lanz, T. 1995, ApJ, 439, 875

İbanoğlu, C. 1978, Ap\&SS, 57, 219

İbanoğlu, C., Evren, S., Taş, G., Çakırlı, Ö. 2005, MNRAS, 360, 1077

Isles, J.E., 1976, Journal of the British Astronomical Association, 86, 412

Jameson, R.F., King, A.R., Sherrington, M.R. 1981, MNRAS, 195, 235

Jeffries, R., Jackson, K., Briggs, P., Evans, J.P. 2010, MNRAS, 411, 2029

Johnson, J.J., Harrison, T.E., Howell, S.B., Szkody, P., McArthur, B.E., Benedict, G.F. 2003, BAAS, 202, 0702

Kato, T. et al. 2002, A\&A, 396, 929

Kato, T. et al. 2004, MNRAS, 347, 861

Knigge, C. 2006, MNRAS, 373, 484

Knigge, C. 2007, MNRAS, 382, 1982

Kraft, R.P. 1964, 1st Conf. on Faint Blue Stars, ed. Luyten, U. of Minnesota Press, Minneapolis

Kubiak, M., Pojmanski, G., Krzeminski, W. 1999, Acta Astronomica, 49, 73

La Dous, C. 1991, A\&A, 252, 100

Lasota, J.-P. 2001, New Astron. Rev., 45, 449

Lake, J., \& Sion, E.M. 2001, AJ, 122, 1632

Lesniak, M.V., \& Sion, E.M. 2003, AAS, 203, 4412

Linnell, A.P., Szkody, P., Gänsicke, B.T., Long, K.S., Sion, E.M., Hoard, D.W., Hubeny, I. 2005, ApJ, 624, 923

Linnell, A.P., Godon, P., Hubeny, I., Sion, E.M., Szkody, P. 2007, ApJ, 662, 1204

Linnell, A.P., Godon, P., Hubeny, I., Sion, E.M., Szkody, P. \& Barrett, P.E. 2008a, ApJ, 676,1226 
Linnell, A.P., Godon, P., Hubeny, I., Sion, E.M., Szkody, P. 2008b, ApJ, 688, 568

Linnell, A.P., Godon, P., Hubeny, I., Sion, E.M., Szkody, P. \& Barrett, P.E. 2009, ApJ, 703, 1839

Linnell, A.P., Godon, P., Hubeny, I., Sion, E.M., Szkody, P. 2010, ApJ, 719, 271

Long, K.S., Blair, W., Hubeny, I., \& Raymond, J. 1996, ApJ, 468, 871

Long, K.S., Froning, C.S., Gänsicke, B.T., \& Knigge, C. 2007, ASPC, 372, 505

Long, K.S., Froning, C.S., Knigge, C., Blair, W.P., Kallman, T.R., Ko, Y.-K. 2005, ApJ, 630,511

Long, K.S., Gänsicke, B.T., Knigge, C., Froning, C.S., Monard, B. 2009, ApJ, 697, 1512

Long, K.S., Wade, R.A., Blair, W.P., Davidsen, A.F., Hubeny, I. 1994, ApJ, 426, 704

Mateo, M., \& Szkody, P. 1984, AJ, 89, 863

Mauche, C.W. 1991, ApJ, 373, 624

Mauche, C.W. 1996, in A. Evans and J.H. Woods (eds.), Cataclysmic Variables and Related Objects, 243 (Kluwer Academic Publishers, The Netherlands)

Mauche, C.W., Raymond, J.C., \& Córdova, F.A. 1988, ApJ, 335, 829

Mauche, C., Wade, R.A, Polidan, R.S., van der Woerd, H., \& Paerels, F.B.S. 1991, ApJ, 372,659

Meinunger, L. 1979, Inf.Bull.Variable Stars, 1611, 1

Menzies, J.W., Odonoghue, D., Warner, B. 1986, Ap\&SS, 122, 73

Metz, K. 1989, Inf. Bull. Var. Stars, 3385

Mikolajewska, J., Acker, A., \& Stenholm, B. 1997, A\&A, 327, 191

Mikolajewska, J., Belczyński, K., Brandi, E., Garcia, L.G., Ferrer, O.E. 1999, BAAA, 43, 31

Mikolajewska, J., Kenyon, S.J., \& Mikolajewski, M. 1989, AJ, 98, 1427

Mikolajewska, J., Kenyon, S.J., Mikolajewski, M., Garcia, M.R., Polidan, R.S, 1995, AJ, 109,1289 
Mikolajewska, J., Quiroga, C., Brandi, E., Garcia, L.G., Ferrer, O.E., \& Belczyński, K., 2003, in Symbiotic Stars, Probing Stellar Evolution, ed. R.L.M. Corradi, J. Mikolajewska, \& T.J. Mahoney (San Francisco, CA: ASP) 147

Moos, H.W., et al. 2000, ApJ, 538, L1

Morton, D.C. 2000, ApJS, 130, 403

Morton, D.C. 2003, ApJS, 149, 205,

Mukai, K. 2012, online catalog of IPs asd.gsfc.nasa.gov/Koji.Mukai/iphome/iphome.html

Mumford, G.S. 1979, BAAS 11, 403

Mumford, G.S 1980, AJ, 85, 748

Mumford, G.S., \& Krezminski, W. 1969, ApJS, 18, 429

Mürset, U., Nussbaumer, H., Schmid, H.M., \& Vogel, M. 1991, A\&A, 248, 458

North, R.C., Marsh, T.R., Kolb, U., Stehle, R., Smith, R.C., 2000a, NewAR 44, 29

North, R.C., et al. 2000b, MNRAS, 313, 383

North, R.C., Marsh, R.R., Kolb, U., Dhillon, V.S., Moran, C.K.J. 2002, MNRAS, 337, 1215

Notni, P., \& Richter, G.A. 1984, Inf.Bull.Variable Stars No.2634

O’Brien, S., Bond, H.E., Sion, E.M. 2001, ApJ, 563, 971

O'Connell, D.J.K. 1932, Harvard College Observatory Bulletin, 890, 180

Pandel, D., Cordova, F.A., Howell, S.B. 2003, MNRAS, 346, 1231

Panei, J.A., Althaus, L.G., \& Benvenuto, O.G. 2000, A\&A, 353, 970

Patterson, J. 1984, ApJS, 54, 443

Patterson, J. 2002, CBAstro Communication, (March 28;

http://cbastro.org/communications/news/messages/0249.html)

Patterson, J. et al. 2005, PASP, 117, 1204

Patterson, J., Thorstensen, J.R., \& Kemp, J. 2005, PASP, 117, 427

Perryman, M.A.C., et al. 1997, A\&A, 323L, 49 
Polidan, R.S., \& Carone, T.E., 1986, BAAS 18, 1016

Polidan, R.S., Mauche, C.W., \& Wade, R.A. 1990, ApJ, 356, 211

Press, W.H., Teukolsky, S.A., Vetterling, W.T., Flannery, B.P., Numerical Recipes in Fortran 77, The Art of Scientific Computing, Second Edition, 1992, Cambridge University Press

Pretorius, M.L., Warner, B., \& Woudt, P.A. 2006, MNRAS, 368, 361

Pringle, J. 1975, MNRAS, 170, 633

Prinja, R.K., \& Rosen, R. 1995, MNRAS, 273, 461

Prinja, R.K., Long, K.S., Froning, C.S., Knigge, C., Witherick, D.K., Clark, J.S., \& Ringwald, F.A. 2003, MNRAS, 340, 551

Provencal, J., Shipman, H.L., Hoeg, E., Thejll, P. 1996, AAS 189.7901

Puebla, R.E., Diaz, M.P., Hubeny, I. 2007, AJ, 134, 1923

Ramseyer, T.F., Hatzes, A.P., \& Jablonski, F. 1995, AJ, 110, 1364

Ribeiro, F.M.A., \& Diaz, M.P. 2007, AJ, 133, 2659

Richter, G.A. 1961, Veroeff.Sternw.Sonneberg, 4, 433

Richter, G.A., Notni, P., Tiersch, H. 1988, Astron.Nach., 309, 91

Ringwald, R.A. 1994, Interacting Binary Stars, ASP Conferences Series, Vol.56, A.W. Shafter (ed.), p. 294

Ringwald, F.A., Thorstensen, J., Honeycutt, R., \& Smith, R.C. 1996, AJ, 111, 2077

Ritter, H., \& Kolb, U. 2003, A\&A, 404, 301

Robinson, E.L. 1974, ApJ, 193, 191

Robinson E.L., Barker, E.S., Cochran, A.L., Cochran, W.D., Nather, R.E. 1981, ApJ, 251, 611

Rodgers, A.W., Roberts, W.H., \& Walker, I. 1993, AJ, 106, 591

Rodgers, A.W., \& Roberts, W.H. 1994, IAUC 6043

Sahnow, D.J. et al. 2000, ApJ, 538, L7 
Sarna, M.J., Pych, W., \& Smith, R.C. 1995, IBVS No. 4165

Schmid, H.M. 1989, A\&A, 211, L31

Schmid, H.M. et al. 1999, A\&A, 348, 950

Schneider, D.P., Young, P., Shectman, S.A. 1981, ApJ, 245, 644

Schoembs, R., \& Vogt, N. 1981, A\&A, 97, 185

Shafter, A. 1983, Ph.D. Thesis, UCLA

Shafter, A. \& Harkness, R.P. 1986, ApJ, 92, 685

Shakun, L.I. 1987, Astronomicheskii Tsirkulyar, 1491, 7

Shakura, N.I., \& Sunyaev, R.A., 1973, A\&A, 24, 337

Shaviv, G. \& Wehrse, R. 1991, A\&A, 251, 117

Sheets, H.A., Thorstensen, J.R., Peters, C.M, Kapusta, A.B., Taylor, C.J., 2007, PASP, 119, 494

Simon, V. 2000, A\&A, 354, 103

Sion, E.M. 1985, ApJ, 292, 601

Sion, E.M. \& Urban, J. 2002, ApJ, 572, 456

Sion, E.M., Bond, H.E., Lindler, D., Godon, P., Wickramasinghe, D., Ferrario, L., \& Dupuis, J. 2012, ApJ, 751, 66

Sion, E.M., Cheng, F., Godon, P., Urban, J.A., Szkody, P. 2004a, AJ, 128, 183

Sion, E.M., Cheng, F.H., Huang, M., Hubeny, I., \& Szkody, P. 1996, ApJ, 471, L41

Sion, E.M., Cheng, F., Long, K.S., Szkody, P., Huang, M., Gilliland, R., \& Hubeny, I. 1995a, ApJ, 439, 957

Sion, E.M., Cheng, F.H., Sparks, W.M., Szkody, P., Huang, M., \& Hubeny, I. 1997, ApJ, 480, L17

Sion, E.M., Gänsicke, B.T., Long, K.S., Szkody, P., Knigge, C., Hubeny, I., de Martino, D., Godon, P., 2008, ApJ, 681, 543

Sion, E.M., Godon, P., Cheng, F., Szkody, P., 2007, AJ, 134, 886 
Sion, E.M., Godon, P., Myszka, J., Blair, W.P., 2010, ApJ, 716, L157

Sion, E.M., Schaeffer, K.G., Bond, H.E., Saffer, R.A., Cheng, F.H. 1998, ApJ, 496, L29

Sion, E.M., Szkody, P., Cheng, F.H., \& Huang, M. 1995b, ApJ, 444, L97

Sion, E.M., Szkody, P., Cheng, F.H., Gänsicke, B., LaDous, C., \& Hassal, B.J.M. 2001, ApJ, 555,834

Sion, E.M., Winter, L., Urban, J.A., Tovmassian, G.H., Zharikov, S., Gänsicke, B.T., Orio, M. 2004b, AJ, 128, 1795

Skillman, D.R., \& Patterson, J. 1988, AJ, 96, 976

Skillman, D.R., Patterson, J., Thorstensen, J.R. 1995, PASP, 107, 545

Skopal, A. 2005, A\&A, 440, 995

Skopal, A., Sekerás, M., González-Riestra, R., Viotti, R.F. 2009, A\&A, 507, 1531

Skopal, A., Vanko, M., Pribulla, T., Chochol, D., Semkov, E., Wolf, M., \& Jones, A. 2007, Astron. Nachr., 328, 909

Smith, V.V., Cunha, K., Jorissen, A., Boffin, H.M.J. 1996, A\&A, 315, 179

Smith, R.C., Sarna, M.J., Catalan, M.S., \& Jones, D.H.P. 1997, MNRAS, 287, 271

Spogli, C., Fiorucci, M., Raimondo, G. 2003a, IBVS 5365, 1

Spogli, C., Fiorucci, M., Dolci, M., Raimondo, G. 2003b, IBVS 5474, 1

Spogli, C., et al. 2005, IBVS 5996, 1

Sproats, L.N., Howell, S.B., \& Mason, K.O. 1996, MNRAS, 282, 1211

Stokes, S.J., Evans, J.M., Bianchini, A., Canterna, R., 2000, AAS, 197.8505

Stover, R. 1981, ApJ, 249, 673

Stover, R.J., Robinson, E.L., Nather, R.E. 1981, ApJ, 248, 696

Stump, M., \& Sion, E.M. 2001, PASP, 1131222

Szkody, P., \& Downes, R.A. 1982, PASP, 94, 328

Szkody, P., \& Mattei, J.A. 1984, PASP, 96, 988 
Szkody, P., Crosa, L., Bothun, G.D., Downes, R.A., \& Schommer, R.A. 1981, ApJ, 249, L61

Szkody, P., Downes, R., \& Mateo, M. 1988, PASP, 100, 362

Tappert, C., Wargau, W., Hanuschik, R.W., \& Vogt, N. 1997, A\&A, 327, 231

Taylor, C.M., \& Thorstensen, J.R. 1996, PASP, 108, 894

Thorstensen, J.R., 1986, AJ, 91, 940

Tomov, N., Tomova, M., Ivanova, A. 2000, A\&A, 364, 557

Tomov, N., Tomova, M. 2002, A\&A, 388, 202

Tovmassian, G., Orion, M., Zharikov, S., Echevarria, J., Costero, R., \& Michel, R. 2002, in AIP Conf.Proc.637, Classical Nova Explosions, ed.M.Hernanz \& J.Jose (Melville, NY: AIP), 72

Urban, J.A., Sion, E.M. 2006, ApJ, 642, 1029

Van Altena, W.F., Lee, J.T., Hoffleit, D.E. 1995, The General Catalog of Trigonometric Parallaxes, 4th Edition, Yale University Observatory

van Amerongen, S., Damen, E., Groot, M., Kraakman, H., \& van Paradijs, J. 1987a, MNRAS, 225, 93

van Amerongen, S., Bovenschen, H., \& van Paradijs, J. 1987b, MNRAS, 229, 245

van Teeseling, A., Drake, J.J., Drew, J.E., Hoare, M.G., Verbunt, F. 1995, A\&A, 300, 808

Verbunt, F. 1987, A\&A, 89, 223

Viotti, R., Altamore, A., Baratta, G.B., Cassatella, A., \& Friedjung, M. 1984, ApJ, 283, 226

Viotti, R., Ricciardi, O., Giangrande, A., Ponz, D., Friedjung, M., Cassatella, A., Baratta, G.B., Altamore, A. 1983, A\&A, 119, 285

Vogt, N., and Bateson, F.M. 1982, A\&AS, 48, 383

Vogt, N. \& Breysacher, J. 1980, ApJ, 235, 945

Vogt, N. \& Semeniuk, I. 1980, A\&A, 89, 223

Wade, R. 1982, AJ, 87, 1558

Wade, R.A. 1988, ApJ, 335, 394 
Wade, R.A., \& Hubeny, I. 1998, ApJ, 509, 350

Walter, F. 2003, in the Future of Cool-Star Astrophysics: 12th Cambridge Workshop on Cool Stars, Stellar Systems, and the Sun, eds. A. Brown, G.M. Harper, T.R. Ayres (University of Colorado), 14

Walter, F. 2004, A.N., 325, 241

Wargau, W., Drechsel, H., Rahe, J., Bruch, A. 1983, MNRAS, 204, 35P

Wargau, W., Drechsel, H., Rahe, J., Bruch, A., Schombs, R. 1984, Ap\&SS, 99, 145

Warner, B. 1982, Inf.Bul.Var.Stars, No.2175

Warner, B., Odonoghue, D., Allen, S. 1985, MNRAS, 212, 9

Warner, B. 1987, MNRAS, 227, 23

Warner, B. 1995, Cataclysmic Variable Stars (Cambridge: Cambridge Univ. Press)

Watson, C.A., Steeghs, D., Shahbaz, T., Dhillon, V.S. 2007a, MNRAS, 382, 1105

Watson, C.A., Steeghs, D., Dhillon, V.S., Shahbaz, T. 2007b, Astron.Nachr., 328, 813

Welsh, W.F., Froning, C.S., Marsh, T.R., Robinson, E.L., Wood, J.H., 2005, ASPC 330, 351

Welsh, W.F., Froning, C.S., Marsh, T.R., Reimer, T.W., Robinson, E.L., Wood, J.P.R. 2007, ASPC 362, 241

Werner, K., \& Rauch, R. 1997, A\&A, 324, L25

Wheatley, P.J. 1998, MNRAS, 297, 1145

Wheatley, P.J., Verbunt, F., Belloni, T., Watson, M.G., Naylor, T., Ishida, M., Duck, S.R., Pfeffermann, E. 1996, A\&A, 307, 137

Williams, G., 1983, ApJS, 53, 523

Williger, G., Berriman, G., Wade, R.A., Hassall, B.J.M. 1988, ApJ, 333, 277

Winter, L. \& Sion, E.M. 2001, BAAS, 33, 1399

Winter, L. \& Sion, E.M. 2003, ApJ, 582, 352

Woods, J.A., Verbunt, F., Collier Cameron, A., Drew, J.E., \& Piters, A. 1992, MNRAS, 255,237 
Wood, M.A. 1995, in White Dwarfs, Proceedings of the 9th European Workshop on White Dwarfs, Lecture Notes in Physics, Vol.442, eds. Detlev Koester \& Klaus Werner, Springer-Verlag, Berlin Heidelberg New York, p.41

Young, P., \& Schneider, D.P. 1981, ApJ, 247, 960

Young, A., \& Nelson, B. 1972, ApJ, 173, 653

Young, A., Klimke, A., Africano, J.L., Quigley, R., Radick, R.R., van Buren, D. 1983, ApJ, 267,655

Young, P.R., Dupree, A., Espey, B.R., Kenyon, S.J., Ake, T.B. 2005, ApJ, 618, 891

Zacharias, N., Urban, S.E., Zacharias, M.I., Wycoff, G.L., Hall, D.M., Monet, D.G., Rafferty, T.J. 2004, AJ, 127, 3043

Zhang, E., 1989, Publications of the Beijing Astronomical Observatory, 13, 37 
Table 1. Adopted System Parameters

\begin{tabular}{|c|c|c|c|c|c|c|c|c|}
\hline $\begin{array}{l}\text { System } \\
\text { Name }\end{array}$ & $\begin{array}{l}\text { RA } \\
\text { hh:mm:ss }\end{array}$ & $\begin{array}{r}\text { DEC } \\
\text { deg:mm:ss }\end{array}$ & $\begin{array}{l}\text { Type/ } \\
\text { Subtype }\end{array}$ & $\begin{array}{c}\mathrm{P} \\
(\mathrm{hrs})\end{array}$ & $\begin{array}{c}\mathrm{i} \\
(\mathrm{deg})\end{array}$ & $\mathrm{E}(\mathrm{B}-\mathrm{V})$ & $\begin{array}{l}M_{w d} \\
\left(M_{\odot}\right)\end{array}$ & $\begin{array}{c}\mathrm{d} \\
(\mathrm{pc})\end{array}$ \\
\hline AE Aqr & 20:40:09.07 & $-00: 52: 16.3$ & $\mathrm{Mg}$ IP & 9.88 & 66 & $\ldots$ & 0.63 & 102 \\
\hline AE Ara & $17: 41: 04.91$ & $-47: 03: 27.3$ & Msc R & $\ldots$ & $\ldots$ & $\ldots$ & $\ldots$ & $\ldots$ \\
\hline AG Dra & $16: 01: 41.01$ & $+66: 48: 10.1$ & Msc R & $\ldots$ & $\ldots$ & $\ldots$ & $\ldots$ & $\ldots$ \\
\hline AM Cas & $02: 26: 23.45$ & $+71: 18: 31.4$ & $\mathrm{DN} \mathrm{ZC}$ & 3.96 & 18 & 0.20 & 0.55 & 350 \\
\hline AM Her & $18: 16: 13.33$ & $+49: 52: 04.2$ & Mg P & 3.09425 & 50 & $\ldots$ & 0.76 & 79 \\
\hline AQ Men & 05:07:53.90 & $-79: 51.22 .9$ & DN UG & 3.40 & $\ldots$ & $\ldots$ & $\ldots$ & 710 \\
\hline BB Dor & $05: 29: 28.59$ & $-58: 54: 46.3$ & NL VY & 3.58 & 08 & 0.00 & $\ldots$ & $\ldots$ \\
\hline BV Cen & $13: 31: 18.60$ & $-54: 58: 32.0$ & DN UG & 14.66830 & 53 & 0.10 & 1.20 & 238 \\
\hline $\mathrm{CH}$ UMa & 10:07:00.77 & $+67: 32: 48.7$ & DN UG & 8.23642 & 21 & 0.00 & 1.35 & 314 \\
\hline DQ Her & 18:07:30.12 & $+45: 51: 32.7$ & $\mathrm{Mg}$ IP & 4.65 & 90 & $\ldots$ & 0.60 & 525 \\
\hline DT Aps & $17: 22: 47.74$ & $-75: 09: 56.6$ & DN SS & $\ldots$ & $\ldots$ & $\ldots$ & $\ldots$ & 710 \\
\hline EK TrA & 15:14:01.47 & $-65: 05: 31.3$ & DN SU & 1.5091 & 58 & 0.03 & 0.46 & $\ldots$ \\
\hline EM Cyg & 19:38:40.01 & $+30: 30: 27.0$ & DN ZC & 6.98182 & 67 & 0.05 & 1.00 & 400 \\
\hline ES Dra & $15: 25: 31.68$ & $+62: 01: 00.2$ & DN UG & 4.29 & $\ldots$ & 0.00 & $\ldots$ & $\ldots$ \\
\hline EX Hya & $12: 52: 24.47$ & $-29: 14: 57.5$ & $\mathrm{Mg}$ IP & 1.63761 & 78 & $\ldots$ & 0.79 & 65 \\
\hline EY Cyg & $19: 54: 36.77$ & $+32: 21: 54.7$ & DN UG & 11.02377 & 16 & 0.00 & 1.26 & $\ldots$ \\
\hline FO Per & 04:08:34.99 & $+51: 14: 48.3$ & DN UG & 4. & $\ldots$ & $\ldots$ & $\ldots$ & 270 \\
\hline HP Nor & $16: 20: 49.58$ & $-54: 53: 23.0$ & DN ZC & $\ldots$ & $\ldots$ & $\ldots$ & $\ldots$ & $\ldots$ \\
\hline IX Vel & $08: 15: 18.97$ & $-49: 13: 20.7$ & NL UX & 4.65425 & 57 & $\ldots$ & 0.82 & 96 \\
\hline MU Cam & $06: 25: 16.23$ & $+73: 34: 38.9$ & Mg IP & 4.71864 & $\ldots$ & $\ldots$ & $\ldots$ & $\ldots$ \\
\hline MV Lyr & 19:07:16.30 & $+44: 01: 08.4$ & NL VY & 3.19 & 12 & 0.00 & 0.73 & 550 \\
\hline NSV 10934 & $18: 40: 52.45$ & $-83: 43: 09.7$ & DN SU & 1.74 & $\ldots$ & $\ldots$ & $\ldots$ & 150 \\
\hline P831 57 & $03: 34: 34.20$ & $-64: 00: 56.3$ & $\mathrm{MSc}$ & $\ldots$ & $\ldots$ & $\ldots$ & 0.55 & 115 \\
\hline RU Peg & $22: 14: 02.58$ & $+12: 42: 11.4$ & DN UG & 8.9904 & 33 & 0.00 & 1.29 & 282 \\
\hline RW Sex & 10:19:56.62 & $-08: 41: 56.1$ & NL UX & 5.88168 & 34 & 0.01 & 0.8 & 150 \\
\hline SS Aur & $06: 13: 22.40$ & $+47: 44: 26.1$ & DN UG & 4.3872 & 38 & 0.08 & 1.08 & 201 \\
\hline SS Cyg & $21: 42: 42.70$ & $+43: 35: 09.5$ & DN UG & 6.60312 & 51 & 0.04 & 0.81 & 166 \\
\hline TX Col & $05: 43: 20.27$ & $-41: 01: 56.1$ & $\mathrm{Mg}$ IP & 5.718 & $\ldots$ & $\ldots$ & 0.54 & $\ldots$ \\
\hline UU Aql & $19: 57: 18.76$ & $-09: 19: 20.9$ & DN UG & 3.92477 & $\ldots$ & $\ldots$ & $\ldots$ & 380 \\
\hline V3885 Sgr & $19: 47: 40.53$ & $-42: 00: 26.4$ & NL UX & 4.97186 & 65 & 0.02 & 0.70 & 110 \\
\hline V405 Aur & $05: 57: 59.27$ & $+53: 53: 45.1$ & $\mathrm{Mg}$ IP & 4.16 & 04 & $\ldots$ & $\ldots$ & $\ldots$ \\
\hline V471 Tau & $03: 50: 24.97$ & $+17: 14: 47.4$ & Msc PCV & 12.50839 & 79 & 0.00 & 0.83 & 47 \\
\hline V794 Aql & $20: 17: 34.03$ & $-03: 39: 50.2$ & NL VY & 3.68 & 60 & 0.20 & 0.88 & $\ldots$ \\
\hline VW Hyi & 04:09:11.34 & $-71: 17: 41.1$ & DN SU & 1.783 & 60 & 0.01 & 0.86 & 65 \\
\hline WW Cet & 00:11:24.80 & $-11: 28: 44.1$ & DN UG & 4.22 & 54 & 0.00 & 0.85 & 200 \\
\hline YY Dra & $11: 43: 38.51$ & $+71: 41: 19.2$ & Mg IP & 3.96 & 42 & & 0.83 & 155 \\
\hline
\end{tabular}

Note: the systems are listed in alphabetical order of the first letters, rather than of the constellation, since this is the way the systems are returned by the catalog search page. 
Table 2. FUSE Observation Log

\begin{tabular}{|c|c|c|c|c|c|}
\hline $\begin{array}{l}\text { System } \\
\text { Name }\end{array}$ & DATAID & $\begin{array}{c}\text { Obs. date } \\
\text { yyyy-mm-dd }\end{array}$ & $\begin{array}{c}\text { Obs. time (UT) } \\
\text { hh:mm:ss }\end{array}$ & $\begin{array}{l}\text { Exp.time } \\
\text { sec }\end{array}$ & state \\
\hline AE Aqr & B0340101 & 2001-06-17 & $12: 26: 34$ & 28821 & \\
\hline AE Ara & D1460201 & 2004-05-18 & $17: 41: 56$ & 9831 & \\
\hline AG Dra & S3120102 & 2000-03-16 & $15: 57: 00$ & 2387 & \\
\hline AM Cas & G9251402 & 2006-10-19 & $03: 17: 49$ & 12909 & intermediate \\
\hline AM Her & Z0060101 & 2002-05-11 & $23: 39: 59$ & 46901 & low \\
\hline AQ Men & G9250201 & 2006-11-22 & $16: 16: 28$ & 22661 & intermediate \\
\hline BB Dor & H9030301 & 2007-07-09 & 02:51:09 & 3500 & high \\
\hline BV Cen & D1450301 & 2003-04-13 & $20: 26: 00$ & 26545 & low \\
\hline $\mathrm{CH}$ UMa & D1450201 & 2003-04-02 & 22:00:00 & 17311 & low \\
\hline DQ Her & D9130501 & 2003-06-30 & $13: 20: 51$ & 7355 & \\
\hline DR Aps & G9251101 & 2006-04-28 & $10: 47: 43$ & 68216 & intermediate \\
\hline EK TrA & Z9104301 & 2002-06-24 & $07: 22: 05$ & 33525 & low \\
\hline EK TrA & Z9104302 & 2002-06-24 & $20: 39: 39$ & 33842 & low \\
\hline EK TrA & combined & 2002-06-24 & $07: 22: 05$ & 67367 & low \\
\hline EM Cyg & C0100101 & 2002-09-05 & $11: 34: 11$ & 1479 & low \\
\hline ES Dra & G9251601 & 2006-11-19 & $23: 08: 35$ & 24554 & intermediate \\
\hline EX Hya & A0840102 & 2000-05-19 & $01: 19: 36$ & 9913 & \\
\hline EY Cyg & D1450101 & 2003-07-16 & $17: 35: 26$ & 19295 & low \\
\hline FO Per & G9251501 & 2007-02-11 & 03:02:06 & 2716 & high \\
\hline HP Nor & G9250601 & 2007-04-13 & $17: 13: 23$ & 3952 & intermediate \\
\hline IX Vel & Q1120101 & 2000-04-15 & $15: 34: 47$ & 6086 & high \\
\hline MU Cam & E9890801 & 2004-11-06 & $06: 47: 54$ & 13909 & \\
\hline MV Lyr & C0410301 & 2002-07-07 & $11: 56: 39$ & 11209 & low \\
\hline NSV10934 & G9251201 & 2006-06-28 & $14: 08: 26$ & 10844 & low \\
\hline NSV10934 & G9251202 & 2006-06-30 & $17: 46: 04$ & 14177 & low \\
\hline NSV10934 & combined & 2006-06-28 & $14: 08: 26$ & 25021 & low \\
\hline P83157 & D9131401 & 2003-11-03 & $02: 26: 19$ & 11865 & \\
\hline RU Peg & C1100101 & 2002-07-04 & $17: 10: 03$ & 3026 & low \\
\hline RW Sex & B1040101 & 2001-05-13 & $14: 48: 05$ & 25614 & high \\
\hline SS Aur & C1100201 & 2002-02-13 & $06: 59: 53$ & 12733 & low \\
\hline SS Cyg & P2420101 & 2001-09-04 & $08: 22: 31$ & 18583 & low \\
\hline RX Col & D9050201 & $2003-12-31$ & $07: 00: 22$ & 3330 & \\
\hline UU Aql & C1100301 & 2004-05-16 & $13: 48: 00$ & 16121 & low \\
\hline V3885 Sgr & P1870101 & 2000-05-24 & $01: 51: 55$ & 12392 & high \\
\hline V405 Aur & D0800101 & 2003-10-01 & 02:06:07 & 21581 & \\
\hline V471 Tau & M1124002 & 2001-01-12 & $05: 27: 23$ & 2145 & \\
\hline V794 Aql & D1440101 & 2004-05-13 & $19: 50: 22$ & 13438 & high \\
\hline VW Hyi & В0700201 & 2001-08-18 & $10: 29: 41$ & 17435 & low \\
\hline WW Cet & D1450401 & $2003-07-26$ & 05:30:03 & 16291 & low \\
\hline YY Dra & C0410201 & $2002-01-30$ & $21: 29: 16$ & 15139 & \\
\hline
\end{tabular}

Note: the systems are listed in alphabetical order of the first letters, rather than of the constellation, since this is the way the systems are returned by the catalog search page. 
Table 3. Lines in FUSE Spectra of Cataclysmic Variables and Related Objects

\begin{tabular}{|c|c|}
\hline Ion & $\lambda(\AA)$ \\
\hline He II $($ Balmer $\mathrm{n}=20)$ & 920.56 \\
\hline N IV & $921.46,921.99,922.52,923.06,923.22,923.68,924.28,924.91$ \\
\hline He II (Balmer $\mathrm{n}=18$ ) & 922.75 \\
\hline He II (Balmer $\mathrm{n}=17$ ) & 924.15 \\
\hline He II (Balmer $\mathrm{n}=15)$ & 927.85 \\
\hline He II (Balmer $\mathrm{n}=14)$ & 930.34 \\
\hline He II (Balmer $\mathrm{n}=13$ ) & 933.45 \\
\hline S VI & 933.38 \\
\hline He II (Balmer $\mathrm{n}=12$ ) & 937.39 \\
\hline He II (Balmer $\mathrm{n}=11$ ) & 942.51 \\
\hline S VI & 944.52 \\
\hline He II (Balmer $\mathrm{n}=10)$ & 949.33 \\
\hline P IV & 950.66 \\
\hline He II (Balmer $\mathrm{n}=9)$ & 958.70 \\
\hline $\mathrm{P}_{\mathrm{II}}$ & $961.04,962.12,962.57,963.62,963.80,964.95,965.40$ \\
\hline Ne VII & 973.35 \\
\hline $\mathrm{C}$ III & 977.02 \\
\hline N III & $989.79 \& 991.56$ \\
\hline He II (Balmer $\mathrm{n}=7$ ) & 992.36 \\
\hline $\mathrm{NeVI}$ & 992.73 \\
\hline Ne VI & 997.17 \\
\hline Ne vi & 999.29 \\
\hline $\mathrm{Ne}$ VI & 1005.79 \\
\hline S IV & 1006.39 \\
\hline $\mathrm{C}_{\text {II }}$ & $1009.86,1010.08,1010.37$ \\
\hline $\mathrm{Ne}$ VI & 1010.32 \\
\hline S III & $1015.50,1015.57,1015.78$ \\
\hline S III & $1021.11,1021.32$ \\
\hline O vi & 1031.93 \\
\hline O vi & 1037.62 \\
\hline S IV & 1062.66 \\
\hline Si IV & $1066.61,1066.64,1066.65$ \\
\hline Fe III & 1066.20 \\
\hline S IV & $1072.98 \& 1073.52$ \\
\hline He II (Balmer $\mathrm{n}=5)$ & 1084.94 \\
\hline S IV & $1098.36,1098.93,1099.48,1100.05$ \\
\hline Si III & $1108.36,1109.94,1113.2$ \\
\hline $\mathrm{P}_{\mathrm{V}}$ & 1117.98 \\
\hline P IV & 1118.55 \\
\hline S II & $1124.40,1124.99$ \\
\hline $\mathrm{PV}$ & 1128.01 \\
\hline Si IV & 1122.49 \\
\hline Si IV & 1128.33 \\
\hline $\mathrm{Ne} v$ & 1136.53 \\
\hline Fe III & 1142.96 \\
\hline Si III & $1140.55,1141.58,1142.29,1144.31,1144.96,1145.67$ \\
\hline
\end{tabular}


Table 3-Continued

\begin{tabular}{ll}
\hline \hline Ion & $\lambda(\AA)$ \\
\hline Ne V & 1145.62 \\
Si III & $1155.00,1155.96,1156.78,1158.10,1160.25,1161.58$ \\
C III & $1174.61,1174.93,1175.26,1175.59,1175.71,1175.99,1176.10,1176.37,1176.77$ \\
\hline
\end{tabular}


Table 4. Main Line Identifications

\begin{tabular}{|c|c|c|c|c|c|c|c|c|c|c|c|c|c|c|c|c|c|}
\hline $\begin{array}{l}\text { System } \\
\text { Name }\end{array}$ & $\begin{array}{l}\text { N IV } \\
923\end{array}$ & $\begin{array}{l}\text { S VI } \\
933 \\
944\end{array}$ & $\begin{array}{l}\mathrm{C}_{\text {III }} \\
977\end{array}$ & $\begin{array}{c}\mathrm{N} \text { III + He II } \\
990\end{array}$ & $\begin{array}{c}\text { S IV } \\
1006\end{array}$ & $\begin{array}{c}\text { C II } \\
1010\end{array}$ & $\begin{array}{c}\text { O VI } \\
1032 \\
1038\end{array}$ & $\begin{array}{c}\text { S IV } \\
1063 \\
1073\end{array}$ & $\begin{array}{l}\text { Si IV } \\
1066\end{array}$ & $\begin{array}{l}\text { He II } \\
1085\end{array}$ & $\begin{array}{c}\text { S IV } \\
1100\end{array}$ & $\begin{array}{l}\mathrm{Si} \text { III } \\
1110\end{array}$ & $\begin{array}{c}\mathrm{PV} \\
1118\end{array}$ & $\begin{array}{l}\text { Si IV } \\
1123\end{array}$ & $\begin{array}{c}\mathrm{P} \text { V }+ \text { Si IV } \\
1128\end{array}$ & $\begin{array}{l}\text { Si III } \\
1143 \\
1158\end{array}$ & $\begin{array}{r}\text { C III } \\
1175\end{array}$ \\
\hline AE Aqr & A & $\mathrm{D}, \mathrm{D}$ & $\mathrm{C}$ & B & D & D & $\mathrm{A}, \mathrm{A}$ & $\mathrm{C}, \mathrm{B}$ & D & A & D & B & D & D & $\mathrm{D}$ & $\mathrm{D}, \mathrm{D}$ & $\mathrm{C}$ \\
\hline AE Ara & $\mathrm{C}$ & $\mathrm{C}, \mathrm{C}$ & $\mathrm{C}$ & D & $\mathrm{C}$ & D & $\mathrm{B}, \mathrm{B}$ & $\mathrm{D}, \mathrm{B}$ & $\mathrm{D}$ & $\mathrm{C}$ & D & D & D & $\mathrm{D}$ & D & $\mathrm{D}, \mathrm{D}$ & B \\
\hline AG Dra & D & $\mathrm{B}, \mathrm{A}$ & A & $\mathrm{C}, \mathrm{A}$ & $\mathrm{D}$ & D & $\mathrm{A}, \mathrm{A}$ & $\mathrm{C}, \mathrm{A}$ & D & A & D & $\mathrm{D}$ & $\mathrm{D}$ & $\mathrm{D}$ & $\mathrm{D}$ & $\mathrm{D}, \mathrm{D}$ & $\mathrm{D}$ \\
\hline AM Cas & $\mathrm{C}$ & $\mathrm{C}, \mathrm{C}$ & $\mathrm{C}$ & $\mathrm{C}, \mathrm{C}$ & D & D & $\mathrm{C}, \mathrm{D}$ & $\mathrm{D}, \mathrm{D}$ & D & $\mathrm{C}$ & $\mathrm{C}$ & $\mathrm{C}$ & D & $\mathrm{C}$ & $\mathrm{C}$ & $\mathrm{D}, \mathrm{D}$ & $\mathrm{D}$ \\
\hline AM Her & D & $\mathrm{D}, \mathrm{D}$ & B & $\mathrm{D}$ & D & D & $\mathrm{C}, \mathrm{C}$ & $\mathrm{D}, \mathrm{D}$ & $\mathrm{D}$ & $\mathrm{C}$ & D & D & D & $\mathrm{D}$ & D & $\mathrm{D}, \mathrm{D}$ & D \\
\hline AQ Men & D & D,D & D & $\mathrm{C}$ & D & D & $\mathrm{D}, \mathrm{D}$ & $\mathrm{C}, \mathrm{C}$ & $\mathrm{D}$ & $\mathrm{D}$ & $\mathrm{C}$ & $\mathrm{C}$ & $\mathrm{C}$ & $\mathrm{C}$ & $\mathrm{C}$ & $\mathrm{C}, \mathrm{C}$ & $\mathrm{C}$ \\
\hline BB Dor & D & $\mathrm{C}, \mathrm{C}$ & $\mathrm{C}$ & D & D & $\mathrm{C}$ & $\mathrm{A}, \mathrm{A}$ & $\mathrm{B}, \mathrm{B}$ & $\mathrm{C}$ & D & $\mathrm{D}$ & B & D & A & A & $\mathrm{C}, \mathrm{D}$ & B \\
\hline BV Cen & D & C,D & B & D & D & $\mathrm{C}$ & $\mathrm{C}, \mathrm{D}$ & $\mathrm{D}, \mathrm{C}$ & $\mathrm{D}$ & D & D & $\mathrm{C}$ & D & A & A & $\mathrm{D}, \mathrm{D}$ & $\mathrm{C}$ \\
\hline CH UMa & $\mathrm{C}$ & $\mathrm{D}, \mathrm{C}$ & $\mathrm{C}$ & D & D & D & $\mathrm{A}, \mathrm{B}$ & $\mathrm{D}, \mathrm{C}$ & D & $\mathrm{C}$ & $\mathrm{D}$ & $\mathrm{D}$ & $\mathrm{C}$ & D & D & $\mathrm{D}$ & A \\
\hline DQ Her & B & $\mathrm{B}, \mathrm{B}$ & A & $\mathrm{B}, \mathrm{B}$ & D & D & $\mathrm{A}, \mathrm{A}$ & $\mathrm{A}, \mathrm{A}$ & $\mathrm{D}$ & A & $\mathrm{C}$ & $\mathrm{C}$ & B & $\mathrm{D}$ & B & $\mathrm{D}, \mathrm{D}$ & A \\
\hline DT Aps & D & $\mathrm{C}$ & $\mathrm{C}$ & $\mathrm{C}$ & D & D & $\mathrm{D}, \mathrm{D}$ & $\mathrm{C}, \mathrm{D}$ & D & D & $\mathrm{D}$ & $\mathrm{C}$ & D & $\mathrm{C}$ & $\mathrm{C}$ & $\mathrm{D}, \mathrm{D}$ & $\mathrm{C}$ \\
\hline EK TrA & D & $\mathrm{D}$ & $\mathrm{C}$ & $\mathrm{C}$ & D & D & $\mathrm{C}, \mathrm{C}$ & $\mathrm{C}, \mathrm{D}$ & $\mathrm{C}$ & D & $\mathrm{D}$ & B & D & B & $\mathrm{D}, \mathrm{B}$ & $\mathrm{D}, \mathrm{D}$ & $\mathrm{C}$ \\
\hline EM Cyg & D & $\mathrm{D}, \mathrm{C}$ & $\mathrm{C}$ & D & D & D & $\mathrm{B}, \mathrm{B}$ & $\mathrm{C}, \mathrm{A}$ & $\mathrm{D}$ & D & $\mathrm{C}$ & B & D & B & B & $\mathrm{D}, \mathrm{D}$ & $\mathrm{C}$ \\
\hline ES Dra & D & $\mathrm{C}, \mathrm{C}$ & $\mathrm{C}$ & $\mathrm{C}$ & B & B & $\mathrm{C}, \mathrm{C}$ & $\mathrm{D}, \mathrm{D}$ & $\mathrm{C}$ & D & B & B & D & B & B & $\mathrm{C}, \mathrm{D}$ & $\mathrm{C}$ \\
\hline EX Hya & D & $\mathrm{A}, \mathrm{A}$ & A & $\mathrm{A}, \mathrm{A}$ & D & A & $\mathrm{A}, \mathrm{A}$ & $\mathrm{A}, \mathrm{A}$ & A & $\mathrm{C}$ & D & A & D & A & B & $\mathrm{D}, \mathrm{D}$ & A \\
\hline EY Cyg & D & $\mathrm{D}, \mathrm{D}$ & D & $\mathrm{C}$ & D & D & $\mathrm{C}, \mathrm{C}$ & $\mathrm{D}, \mathrm{C}$ & D & $\mathrm{D}$ & $\mathrm{D}$ & $\mathrm{C}$ & D & D & $\mathrm{C}$ & $\mathrm{C}, \mathrm{D}$ & $\mathrm{D}$ \\
\hline FO Per & D & $\mathrm{C}, \mathrm{C}$ & B & D & D & D & $\mathrm{C}, \mathrm{C}$ & $\mathrm{D}, \mathrm{C}$ & $\mathrm{C}$ & D & D & B & $\mathrm{C}$ & $\mathrm{C}$ & $\mathrm{C}$ & $\mathrm{C}, \mathrm{D}$ & B \\
\hline HP Nor & D & $\mathrm{C}, \mathrm{C}$ & $\mathrm{C}$ & $\mathrm{C}$ & D & D & $\mathrm{D}, \mathrm{D}$ & $\mathrm{D}, \mathrm{C}$ & $\mathrm{D}$ & D & $\mathrm{D}$ & $\mathrm{C}$ & D & $\mathrm{C}$ & D & $\mathrm{D}, \mathrm{D}$ & $\mathrm{C}$ \\
\hline IX Vel & A & $\mathrm{A}, \mathrm{A}$ & A & B & D & D & $\mathrm{B}, \mathrm{B}$ & $\mathrm{A}, \mathrm{A}$ & $\mathrm{D}$ & D & $\mathrm{D}$ & D & B & $\mathrm{D}$ & B & $\mathrm{D}, \mathrm{D}$ & A \\
\hline MU Cam & $\mathrm{C}$ & $\mathrm{C}, \mathrm{C}$ & $\mathrm{C}$ & $\mathrm{C}$ & $\mathrm{D}$ & $\mathrm{C}$ & $\mathrm{B}, \mathrm{C}$ & $\mathrm{C}, \mathrm{D}$ & D & D & D & D & D & $\mathrm{D}$ & D & $\mathrm{D}, \mathrm{D}$ & $\mathrm{D}$ \\
\hline MV Lyr & D & $\mathrm{D}, \mathrm{D}$ & $\mathrm{C}$ & $\mathrm{C}$ & $\mathrm{D}$ & $\mathrm{C}$ & $\mathrm{D}, \mathrm{D}$ & $\mathrm{B}, \mathrm{B}$ & A & B & $\mathrm{D}$ & B & $\mathrm{C}$ & A & A & $\mathrm{D}, \mathrm{D}$ & A \\
\hline NSV 10934 & D & $\mathrm{C}, \mathrm{C}$ & $\mathrm{C}$ & D & D & D & $\mathrm{B}, \mathrm{B}$ & $\mathrm{D}, \mathrm{D}$ & D & $\mathrm{D}$ & D & D & D & D & D & $\mathrm{D}, \mathrm{D}$ & B \\
\hline P831 57 & D & $\mathrm{D}, \mathrm{D}$ & B & B & $\mathrm{C}$ & B & $\mathrm{D}, \mathrm{D}$ & $\mathrm{A}, \mathrm{A}$ & A & B & $\mathrm{D}$ & A & D & A & A & $\mathrm{D}, \mathrm{D}$ & A \\
\hline RU Peg & $\mathrm{C}$ & $\mathrm{C}, \mathrm{C}$ & $\mathrm{C}$ & $\mathrm{B}, \mathrm{B}$ & $\mathrm{C}$ & $\mathrm{C}$ & $\mathrm{B}, \mathrm{B}$ & $\mathrm{C}, \mathrm{A}$ & A & $\mathrm{C}$ & $\mathrm{C}$ & $\mathrm{C}$ & $\mathrm{D}$ & $\mathrm{D}$ & $\mathrm{C}$ & $\mathrm{D}, \mathrm{D}$ & B \\
\hline RW Sex & B & $\mathrm{B}, \mathrm{B}$ & B & B & $\mathrm{C}$ & $\mathrm{C}$ & $\mathrm{D}, \mathrm{D}$ & $\mathrm{D}, \mathrm{B}$ & D & $\mathrm{C}$ & D & D & B & B & B & $\mathrm{C}, \mathrm{D}$ & A \\
\hline SS Aur & D & $\mathrm{D}, \mathrm{D}$ & B & $\mathrm{C}$ & D & B & $\mathrm{C}, \mathrm{C}$ & $\mathrm{D}, \mathrm{C}$ & B & $\mathrm{D}$ & D & $\mathrm{C}$ & D & $\mathrm{C}$ & $\mathrm{D}, \mathrm{C}$ & $\mathrm{C}, \mathrm{C}$ & $\mathrm{D}$ \\
\hline SS Cyg & D & $\mathrm{D}, \mathrm{D}$ & A & $\mathrm{C}$ & D & D & $\mathrm{B}, \mathrm{B}$ & $\mathrm{D}, \mathrm{D}$ & $\mathrm{D}$ & $\mathrm{D}$ & D & D & D & D & $\mathrm{D}$ & $\mathrm{D}, \mathrm{D}$ & A \\
\hline TX Col & $\mathrm{C}$ & D,D & D & $\mathrm{C}$ & D & D & $\mathrm{B}, \mathrm{B}$ & $\mathrm{C}, \mathrm{C}$ & $\mathrm{D}$ & $\mathrm{C}$ & D & D & D & $\mathrm{D}$ & $\mathrm{D}$ & $\mathrm{D}, \mathrm{D}$ & $\mathrm{C}$ \\
\hline UU Aql & D & $\mathrm{C}, \mathrm{C}$ & $\mathrm{C}$ & $\mathrm{C}, \mathrm{C}$ & $\mathrm{D}$ & D & $\mathrm{C}, \mathrm{C}$ & $\mathrm{D}, \mathrm{D}$ & $\mathrm{D}$ & $\mathrm{D}$ & $\mathrm{D}$ & $\mathrm{C}$ & D & $\mathrm{C}$ & $\mathrm{C}$ & $\mathrm{D}, \mathrm{C}$ & $\mathrm{D}$ \\
\hline V3885 Sgr & B & $\mathrm{B}, \mathrm{B}$ & B & $\mathrm{C}$ & D & D & $\mathrm{B}, \mathrm{B}$ & $\mathrm{A}, \mathrm{A}$ & D & $\mathrm{D}$ & D & B & A & $\mathrm{C}$ & B & $\mathrm{D}, \mathrm{D}$ & A \\
\hline V405 Aur & D & $\mathrm{B}, \mathrm{B}$ & A & $\mathrm{C}$ & D & D & $\mathrm{A}, \mathrm{C}$ & $\mathrm{D}, \mathrm{D}$ & $\mathrm{D}$ & B & D & D & D & D & D & $\mathrm{D}, \mathrm{D}$ & B \\
\hline V471 Tau & D & $\mathrm{D}, \mathrm{D}$ & $\mathrm{D}$ & D & D & D & $\mathrm{D}, \mathrm{D}$ & $\mathrm{D}, \mathrm{D}$ & $\mathrm{D}$ & $\mathrm{C}$ & D & $\mathrm{C}$ & D & D & $\mathrm{C}$ & $\mathrm{D}, \mathrm{D}$ & $\mathrm{C}$ \\
\hline V794 Aql & $\mathrm{C}$ & $\mathrm{C}, \mathrm{C}$ & $\mathrm{C}$ & $\mathrm{C}$ & D & D & $\mathrm{C}, \mathrm{C}$ & $\mathrm{D}, \mathrm{D}$ & D & $\mathrm{C}$ & D & D & D & $\mathrm{D}$ & D & $\mathrm{D}, \mathrm{D}$ & $\mathrm{D}$ \\
\hline VW Hyi & $\mathrm{D}$ & $\mathrm{D}, \mathrm{D}$ & $\mathrm{C}$ & $\mathrm{C}$ & $\mathrm{D}$ & $\mathrm{D}$ & $\mathrm{C}, \mathrm{C}$ & $\mathrm{D}, \mathrm{D}$ & $\mathrm{C}$ & B & $\mathrm{C}$ & A & D & $\mathrm{C}$ & A & $\mathrm{B}, \mathrm{D}$ & A \\
\hline WW Cet & D & $\mathrm{D}, \mathrm{D}$ & $\mathrm{C}$ & D & $\mathrm{D}$ & D & $\mathrm{C}, \mathrm{C}$ & $\mathrm{D}, \mathrm{D}$ & $\mathrm{D}$ & $\mathrm{C}$ & $\mathrm{C}$ & B & D & $\mathrm{D}$ & $\mathrm{C}$ & $\mathrm{C}, \mathrm{D}$ & $\mathrm{C}$ \\
\hline YY Dra & D & $\mathrm{D}, \mathrm{D}$ & $\mathrm{C}$ & D & D & D & $\mathrm{C}, \mathrm{C}$ & $\mathrm{D}, \mathrm{D}$ & $\mathrm{D}$ & D & D & D & D & $\mathrm{D}$ & D & $\mathrm{D}, \mathrm{D}$ & $\mathrm{C}$ \\
\hline
\end{tabular}

Note: A denotes a line that has been correctly identified with no contamination and a good S/N.

B denotes a line that has been correctly identified but contaminated, blended, and/or with a lower S/N. C denotes a possible line identification but uncertain due to low S/N. D denotes a line that has not been identified and the marking on the graph is for information only, or for comparison with the theoretical spectrum and cannot be relied on. 
Table 5. ISM lines in FUSE Spectra

\begin{tabular}{|c|c|c|c|c|c|c|c|c|c|c|c|c|c|c|}
\hline H I & $\lambda(\AA)$ & | & O I & $\lambda(\AA)$ & 1 & $\mathrm{H}_{2}$ & $\lambda(\AA)$ & $\mathrm{H}_{2}$ & $\lambda(\AA)$ & $\mathrm{H}_{2}$ & $\lambda(\AA)$ & | & Ion & $\lambda(\AA)$ \\
\hline $30 u$ & 912.70 & 1 & $20 u$ & 921.86 & & L16R2 & 933.24 & L10P3 & 987.77 & L5P1 & 1038.16 & & $\mathrm{~N}_{\mathrm{I}}$ & 953.42 \\
\hline $29 \mathrm{u}$ & 912.77 & 1 & $19 u$ & 922.20 & & W4Q3 & 933.58 & W1R2 & 987.97 & L5R2 & 1038.69 & & $\mathrm{~N}_{\mathrm{I}}$ & 953.66 \\
\hline $27 \mathrm{u}$ & 912.92 & | & $17 \mathrm{u}$ & 925.45 & & L15R1 & 939.12 & L9R1 & 992.01 & L5R3 & 1041.16 & & $\mathrm{~N}_{\mathrm{I}}$ & 954.10 \\
\hline $26 \mathrm{u}$ & 913.01 & 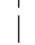 & $16 \mathrm{u}$ & 929.52 & & L15P1 & 939.71 & L9P1 & 992.81 & L5P3 & 1043.50 & & $\mathrm{P}_{\text {II }}$ & 961.04 \\
\hline $25 \mathrm{u}$ & 913.10 & | & $15 \mathrm{u}$ & 930.26 & & W3Q3 & 950.40 & L9R2 & 993.55 & L4R0 & 1049.37 & & $\mathrm{P}_{\text {II }}$ & 963.80 \\
\hline $22 \mathrm{u}$ & 913.48 & | & $12 \mathrm{u}$ & 948.69 & & L13R2 & 956.58 & L9P3 & 997.82 & $\mathrm{~L} 4 \mathrm{R} 2$ & 1051.50 & & Si II/N III & 989.80 \\
\hline $21 \mathrm{u}$ & 913.64 & 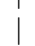 & $11 \mathrm{u}$ & 950.88 & & L13P2 & 957.65 & L8R0 & 1001.82 & $\mathrm{~L} 4 \mathrm{P} 2$ & 1053.28 & & $\mathrm{~S}$ III & 1012.50 \\
\hline $20 u$ & 913.83 & | & $10 u$ & 971.74 & & L13R3 & 958.95 & L8R1 & 1002.45 & L4R3 & 1053.98 & & Si II & 1020.70 \\
\hline $19 \mathrm{u}$ & 914.04 & 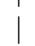 & & 973.23 & & L12R0 & 962.98 & L8P1 & 1003.29 & L4P3 & 1056.47 & & $\mathrm{C}_{\mathrm{II}}$ & 1036.34 \\
\hline $18 \mathrm{u}$ & 914.29 & | & & 973.89 & & L12R1 & 963.61 & L8R2 & 1003.98 & L3R0 & 1062.88 & & $\mathrm{C}_{\text {II }}$ & 1037.02 \\
\hline $17 \mathrm{u}$ & 914.58 & | & $9 \mathrm{u}$ & 972.14 & & $\mathrm{~W} 2 \mathrm{R} 2$ & 965.79 & L8P2 & 1005.39 & L3R1 & 1063.46 & & Ar I & 1048.20 \\
\hline $12 \mathrm{u}$ & 917.18 & | & & 978.62 & & $\mathrm{~W} 2 \mathrm{P} 2$ & 968.29 & W0R3 & 1010.13 & L3P3 & 1070.14 & & Fe II & 1083.42 \\
\hline $11 u$ & 918.13 & | & $5 \mathrm{u}$ & 988.58 & & W2Q3 & 969.05 & W0R2 & 1010.94 & L2R0 & 1077.14 & & $\mathrm{~N}_{\text {II }}$ & 1083.99 \\
\hline $10 \mathrm{u}$ & 919.35 & | & & 988.65 & & $\mathrm{~W} 2 \mathrm{P} 3$ & 970.56 & W0P2 & 1012.17 & L2R1 & 1077.70 & & Fe II & 1096.88 \\
\hline $\mathrm{Ly} \iota$ & 920.96 & | & & 988.77 & & L11P1 & 973.34 & L7R1 & 1013.44 & $\mathrm{~L} 2 \mathrm{P} 1$ & 1078.93 & & Fe II & 1112.05 \\
\hline $\operatorname{Ly} \theta$ & 923.15 & 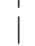 & & 990.13 & 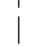 & $\mathrm{L} 11 \mathrm{R} 2$ & 974.16 & L7P1 & 1014.35 & $\mathrm{~L} 2 \mathrm{R} 2$ & 1079.23 & & Fe II & 1121.98 \\
\hline $\mathrm{Ly} \eta$ & 926.23 & | & & 990.80 & & L11P2 & 975.34 & L7R2 & 1014.97 & L1R0 & 1092.20 & & Fe II & 1122.53 \\
\hline $\mathrm{Ly} \zeta$ & 930.75 & 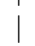 & $4 u$ & 1025.76 & 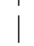 & L10R0 & 981.44 & L7P2 & 1016.46 & L1R1 & 1092.73 & & Fe II & 1125.45 \\
\hline $\mathrm{Ly} \epsilon$ & 937.80 & | & & 1027.43 & & L10R1 & 982.07 & L7R3 & 1017.42 & L1P1 & 1094.05 & & Fe II & 1127.10 \\
\hline Ly $\delta$ & 949.74 & | & & 1028.16 & | & L10P1 & 982.84 & L7P3 & 1019.50 & L1P3 & 1099.79 & & Fe II & 1133.67 \\
\hline $\mathrm{Ly} \gamma$ & 972.54 & | & $3 u$ & 1039.23 & 1 & L10R2 & 983.59 & L6R0 & 1024.37 & L0R0 & 1108.13 & & $\mathrm{~N}_{\mathrm{I}}$ & 1134.17 \\
\hline \multirow[t]{2}{*}{$\mathrm{Ly} \beta$} & 975.00 & | & & 1040.94 & & L10P2 & 984.86 & L6R1 & 1024.99 & L0R1 & 1108.63 & & $\mathrm{~N}_{\mathrm{I}}$ & 1134.42 \\
\hline & & | & & 1041.69 & & $\mathrm{~W} 1 \mathrm{R} 0+1$ & 986.60 & L6R2 & 1026.53 & L0P1 & 1110.06 & & $\mathrm{~N}_{\mathrm{I}}$ & 1134.98 \\
\hline
\end{tabular}

The ISM molecular hydrogen lines are identified by their band (Werner/W or Lyman/L),, upper vibrational level (1-13), and rotational transition ( $\mathrm{R}, \mathrm{P}$, or $\mathrm{Q}$ with lower rotational state $\mathrm{J}=1-3)$. 
Table 6. Accretion Disk \& White Dwarf Synthetic Spectral Model Fits

\begin{tabular}{|c|c|c|c|c|c|c|c|c|c|c|c|c|c|}
\hline $\begin{array}{l}\text { System } \\
\text { Name }\end{array}$ & $\begin{array}{c}\mathrm{i} \\
\operatorname{deg}\end{array}$ & $E_{B-V}$ & $\begin{array}{l}\log (g) \\
\mathrm{cgs}\end{array}$ & $\begin{array}{c}T_{w d} \\
10^{3} \mathrm{~K}\end{array}$ & $\begin{array}{l}V_{\text {rot }} \sin i \\
\mathrm{~km} s^{-1}\end{array}$ & $\begin{array}{c}\mathrm{C} \\
\mathrm{C}_{\odot}\end{array}$ & $\begin{array}{c}\mathrm{Si} \\
\mathrm{Si} \odot\end{array}$ & $\begin{array}{c}\mathrm{S} \\
\mathrm{S} \odot\end{array}$ & $\begin{array}{c}\mathrm{N} \\
\mathrm{N}_{\odot}\end{array}$ & $\begin{array}{c}\mathrm{d} \\
\mathrm{pc}\end{array}$ & $\begin{array}{c}\log (\dot{M}) \\
M_{\odot} / \mathrm{yr}\end{array}$ & model & Reference \\
\hline AM Cas & 18 & 0.00 & 8.25 & 36 & 500 & 1.00 & 1.0 & 1.0 & 1.0 & 373 & -9.7 & wd+disk & Godon et al. (2009a) \\
\hline \multirow[t]{4}{*}{ BB Dor } & $\ldots$ & 0.00 & 8.30 & 37 & 400 & 1.00 & 3.0 & 20. & 1.0 & 211 & $\ldots$ & wd & Godon et al. (2008a) \\
\hline & 8 & 0.00 & 8.30 & $\ldots$ & $\ldots$ & 1.00 & 3.0 & 20. & 1.0 & 665 & -9.0 & disk & $" \quad "$ \\
\hline & 8 & 0.00 & 8.30 & 32 & 400 & 1.00 & 3.0 & 20. & 1.0 & 700 & -9.0 & wd+disk & \\
\hline & 80 & 0.00 & 8.30 & 37 & 400 & 1.00 & 3.0 & 20. & 1.0 & 246 & -9.0 & wd+disk & \\
\hline CH UMa & $\ldots$ & 0.00 & 9.50 & 40 & 200 & $<1.0$ & $<1.0$ & 1.0 & 1.0 & 314 & $\ldots$ & $\mathrm{wd}$ & Sion et al. (2007) \\
\hline EK TrA & $\ldots$ & 0.03 & 8.00 & 17 & 200 & 0.10 & 0.6 & 1.0 & 1.0 & 126 & $\ldots$ & wd & Godon et al. (2008b) \\
\hline EM Cyg & 60 & 0.00 & 8.60 & 50 & 100 & 1.00 & 30.0 & 10. & 10. & 382 & -10.0 & wd+disk & Godon et al. (2009a) \\
\hline \multirow[t]{2}{*}{ ES Dra } & $\ldots$ & 0.00 & 7.8 & 35 & 700 & 1.0 & 10.0 & 50. & 1.0 & 770 & $\ldots$ & wd & Godon et al. (2009a) \\
\hline & 5 & 0.00 & 9.00 & $\ldots$ & $\ldots$ & 1.0 & 10.0 & 50. & 1.0 & 1754 & -9.5 & disk & \\
\hline EY Cyg & $\ldots$ & 0.00 & 9.00 & 30 & 100 & 0.03 & 0.6 & 6.0 & 2.0 & 530 & $\ldots$ & wd & Godon et al. (2008b) \\
\hline \multirow[t]{2}{*}{ FO Per } & 75 & 0.00 & 7.50 & 21 & 200 & 1.00 & 1.0 & 1.0 & 1.0 & 291 & -8.5 & $\mathrm{wd}+$ disk & Godon et al. (2009a) \\
\hline & 18 & 0.30 & 7.50 & 40 & 200 & 1.00 & 1.0 & 1.0 & 1.0 & 254 & -8.5 & wd+disk & \\
\hline P83157 & $\ldots$ & 0.00 & 7.85 & 29 & 001 & 0.01 & 0.02 & 0.01 & 0.01 & 115 & $\ldots$ & wd & Barrett et al. (2012) \\
\hline RU Peg & $\ldots$ & 0.00 & 8.80 & 70 & 40 & 0.20 & 0.2 & 10. & $>1.0$ & 282 & $\ldots$ & wd & Godon et al. (2008b) \\
\hline SS Aur & $\ldots$ & 0.08 & 8.93 & 34 & 400 & 1.00 & 1.0 & 1.0 & 1.0 & 200 & $\ldots$ & wd & Godon et al. (2008b) \\
\hline SS Cyg & 50 & 0.04 & 8.30 & 46 & 200 & 1.0 & 1.0 & 1.0 & 1.0 & 173 & -10.0 & wd+disk & Sion et al. (2010) \\
\hline UU Aql & $\ldots$ & 0.00 & 8.60 & 29 & 200 & 0.001 & 1.0 & 1.0 & 1.0 & 380 & $\ldots$ & wd & Sion et al. (2007) \\
\hline V3885 Sgr & 60 & 0.02 & 8.00 & 60 & 200 & 1.0 & 1.0 & 1.0 & 1.0 & 110 & -8.5 & wd+disk & Linnell et al. (2009), this work \\
\hline V471 Tau & 79 & 0.00 & 8.30 & 33.4 & 250 & 0.0003 & 0.015 & 0.0 & 0.0 & 47 & $\ldots$ & wd & Sion et al. (2012), this work \\
\hline V794 Aql & 60 & 0.20 & 8.60 & $\ldots$ & $\ldots$ & 1.00 & 1.0 & 1.0 & 1.0 & 585 & -8.5 & disk & Godon et al. (2007) \\
\hline VW Hyi & $\ldots$ & 0.01 & 8.00 & 22 & 400 & 0.25 & 1.8 & 1.0 & 3.0 & 60 & $\ldots$ & wd & Godon et al. (2008b) \\
\hline WW Cet & $\ldots$ & 0.00 & 8.35 & 27 & 600 & 0.10 & 0.3 & 1.0 & 2.0 & 213 & $\ldots$ & wd & Godon et al. (2006) \\
\hline
\end{tabular}


Table 7. References

\begin{tabular}{|c|c|}
\hline $\begin{array}{l}\text { System } \\
\text { Name }\end{array}$ & References \\
\hline AE Aqr & see Mukai (2012) and references therein \\
\hline AE Ara & $\begin{array}{l}\text { Cannon (1933); Allen \& Glass (1974); Allen (1978, 1980); Schmid (1989) } \\
\text { Mikola iewska et al. (1989, 1997, 1999, 2003) } \\
\text { Belczyński et al. (2000); Skopal et al. (2007); Fekel et al. (2010) }\end{array}$ \\
\hline AG Dra & $\begin{array}{l}\text { Fekel et al. (2003); Galis et al. (1999); González-Riestra et al. (1999); Greiner et al. (1997) } \\
\text { Meinunger }(1979) ; \text { Mikolaiewska et al. (1995); Mürset et al. (1991); Skopal (2005) } \\
\text { Skopal et al. (2009); Smith et al. }(1996) ; \text { Tomov et al. (2000); Tomov \& Tomova } \\
\text { Schmid et al. (1999); Viotti et al. (1983, 1984); Young et al. (2005) }\end{array}$ \\
\hline AM Cas & $\begin{array}{l}\text { Hoffmeister }(1928) \text {; Richter } \\
\text { Taylor \& Thorstensen (1996); Godon et al. (2009a) }\end{array}$ \\
\hline AM Her & see Gänsicke et al. (2006) and references therein \\
\hline AQ Men & Cheng et al. (2001); Patterson (2002); Godon et al. (2009a) \\
\hline BB Dor & Cheng et al. (2001); Patterson et al. (2005a,b); Godon et al. (2008a) \\
\hline BV Cen & $\begin{array}{l}\text { Vogt \& Brevsacher (1980); Gilliland (1982); Menzies et al. (1986); Williger et al. (1988) } \\
\text { Sion et al. (2007); Watson et al. (2007a,b) }\end{array}$ \\
\hline $\mathrm{CH} \mathrm{UMa}$ & $\begin{array}{l}\text { Becker et al. (1982); Szkody \& Mattei (1984); Thorstensen (1986); Friend et al. (1990) } \\
\text { Simon (2000); Sion et al. (2007) }\end{array}$ \\
\hline DQ Her & see Mukai (2012) and references therein \\
\hline DT Aps & Vogt \& Bateson (1982); Godon et al. (2009a) \\
\hline EK TrA & $\begin{array}{l}\text { Vogt \& Semeniuk (1980); Hassal (1985); Warner (1987); Gänsicke et al. (1997, 2001) } \\
\text { Godon et al. (2008b) }\end{array}$ \\
\hline EM Cyg & $\begin{array}{l}\text { Burbidge \& Burbidge (1953); Kraft (1964); Bever (1967); Mumford \& Krezminski (1969) } \\
\text { Pringle (1975); Robinson (1974); Bengtsson (1975); Brady \& Herczeg (1977) } \\
\text { Mumford (1979); Jameson et al. (1981); Mumford (1980); Bavley (1981) } \\
\text { Stover et al. (1981); Williams (1983); Beuermann \& Pakull (1984); Herczeg (1987) } \\
\text { Hacke (1987); Zhang (1989); Andronov \& Chinarova (1995, 1996) } \\
\text { North et al. (2000a,b, 2002); Winter \& Sion (2003); Spogli et al. (2003a,b, 2005) } \\
\text { Welsh et al. (2005, 2007); Urban \& Sion (2006); Hamilton et al. (2007) } \\
\text { Csizmadia et al. (2008); Godon et al. (2009a) }\end{array}$ \\
\hline ES Dra & $\begin{array}{l}\text { Green et al. } \\
\text { Godon et al. }\end{array}$ \\
\hline EX Hya & see Mukai (2012) and references therein \\
\hline EY Cyg & $\begin{array}{l}\text { Bochkarev \& Sitnik (1985); Sarna et al. (1995); Smith et al. (1997); Costero et al. (1998) } \\
\text { Winter \& Sion (2001); Gänsicke et al. (2003); Tovmassian et al. (2002); Costero et al. (2004) } \\
\text { Sion et al. (2004a); Godon et al. (2008b) }\end{array}$ \\
\hline FO Per & $\begin{array}{l}\text { Howarth (1976); Gessner (1978); Bruch et al. (1987) } \\
\text { Bruch (1989); Sheets et al. (2007); Godon et al. (2009a) }\end{array}$ \\
\hline HP Nor & Vogt \& Bateson (1982); Pretorius et al. (2006); Godon et al. (2009a) \\
\hline IX Vel & $\begin{array}{l}\text { Garrison et al. }(1982,1984) ; \text { Wargau et al. (1983, 1984); Eggen \& Niemela (1984) } \\
\text { Warner (1985); Sion }(1985) ; \text { Haug (1987, 1988); Beuermann \& Thomas (1990) } \\
\text { Polidan et al. (1990); Shaviv \& Wehrse (1991); Mauche (1991); Long et al. (1994) } \\
\text { Prinia \& Rosen (1995); van Teeseling et al. (1995); Kubiak et al. (1999); Hartlev et al. (2002) } \\
\text { Linnell et al. (2007) }\end{array}$ \\
\hline MU Cam & (2012) and $r$ \\
\hline
\end{tabular}


Table 7-Continued

\begin{tabular}{|c|c|}
\hline $\begin{array}{l}\text { System } \\
\text { Name }\end{array}$ & References \\
\hline MV Lyr & $\begin{array}{l}\text { Schneider et al. (1981); Robinson et al. (1981); Chiappetti et al. (1982) } \\
\text { Szkody \& Downes (1982); Skillman et al. (1995); Hoard et al. (2004) } \\
\text { Linnell et al. (2005); Godon \& Sion (2011) }\end{array}$ \\
\hline NSV 10934 & Kato et al. $(2002,2004)$; Godon et al. (2009a) \\
\hline P831-57 & Rodgers et al. (1993); Rodgers \& Roberts (1994); Zacharias et al. (2004); Barrett et al. (2012) \\
\hline RU Peg & $\begin{array}{l}\text { Stover (1981); Wade } \text { (1982); Shafter }(1983) \text {; Friend et al. }(1990) ; \text { Sion \& Urban }(2002) \\
\text { Johnson et al. (2003); Sion et al. (2004); Urban \& Sion (2006); Godon et al. }(2008 \mathrm{~b})\end{array}$ \\
\hline RW Sex & $\begin{array}{l}\text { Greenstein \& Oke }(1982) ; \text { Patterson (1984); Haug \& Drechsel } \\
\text { Polidan \& Carone } 1 \text { (1985); Mauche et al. (1988); Wade (1988); Shaviv \& Wehrse (1991) } \\
\text { Beuermann et al. }(1992) ; \text { Perrvman et al. (1997); Lasota (2001); Prinia et al. (2003) } \\
\text { Linnell et al. (2010); Bolick et al. (1987); Stokes et al. (2000) }\end{array}$ \\
\hline SS Aur & $\begin{array}{l}\text { Shafter (1983); Shafter \& Harkness (1986); Harrison et al. (1999); Lake \& Sion (2001) } \\
\text { Sion et al. (2004); Godon et al. (2008b); Sion et al. (2008) }\end{array}$ \\
\hline SS Cyg & 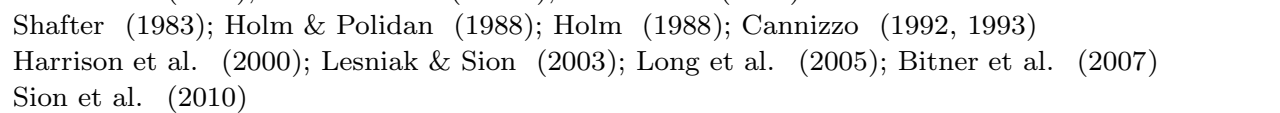 \\
\hline TX Col & see Mukai (2012) and references therein \\
\hline UU Aql & $\begin{array}{l}\text { O'Connell (1932); Isles (1976); Bateson }(1979) ; \text { Davis \& Mattei } \\
\text { Shakun (1987) } \text { (1981) Bateson \& McIntosh (1990); Stump \& Sion (2001) } \\
\text { Harrison et al. (2005); Sion et al. (2007) }\end{array}$ \\
\hline V3885 Sgr & $\begin{array}{l}\text { Guinan \& Sion (1982); Haug \& Drechsel (1985b) } \\
\text { Metz (1989); Woods et al. (1992); Perryman et al. (1997) } \\
\text { Panei et al. (2000); Hartley et al. (2002, 2005); Knigge (2006, 2007) } \\
\text { Ribeiro \& Diaz (2007); Linnell et al. (2009) }\end{array}$ \\
\hline V405 Aur & see Mukai (2012) and references therein \\
\hline V471 Tau & $\begin{array}{l}\text { Young \& Nelson (1972); Cester \& Pucillo (1976); İbanoğlu (1978) } \\
\text { Young et al. (1983); Guinan \& Sion (1984); Skillman \& Patterson (1988) } \\
\text { Bois et al. (1988); Clemens et al. (1992); Barstow et al. (1992) } \\
\text { Ramsever et al. (1995); Van Altena et al. (1995); Provencal et al. (1996) } \\
\text { Dupuis et al. (1997); Barstow et al. (1997); Werner \& Rauch (1997) } \\
\text { Wheatley (1998); Sion et al. (1998); O'Brien et al. (2001); Bond et al. (2001) } \\
\text { Walter (2003, 2004); İbanoğlu et al. (2005); Jeffries et al. (2010) } \\
\text { Sion et al. (2012) }\end{array}$ \\
\hline V794 Aql & $\begin{array}{l}\text { Szkody et al. (1981, 1988); Warner (1982); Honevcutt \& Schlegel (1985) } \\
\text { Honevcutt et al. (1994); Honevcutt \& Robertson (1998); Godon et al. (2007) }\end{array}$ \\
\hline VW Hyi & $\begin{array}{l}\text { Schoembs \& Vogt (1981); Mateo \& Szkodv (1984); van Amerongen et al. (1987a,b) } \\
\text { Warner (1987); Polidan et al. (1990); Belloni et al. (1991); Mauche et al. (1991) } \\
\text { Sion et al. (1995a,b, 1996); Huang et al. (1996a,b); Long et al. (1996) } \\
\text { Mauche (1996); Wheatley et al. (1996); Gänsicke \& Beuermann }(1996) \\
\text { Sion et al. (1997, 2001); Pandel et al. (2003); Long et al. (2007) } \\
\text { Godon et al. (2004); Godon \& Sion (2005); Godon et al. (2008b); Long et al. (2009) }\end{array}$ \\
\hline WW Cet & $\begin{array}{l}\text { Young \& Schneider }(1981) ; \text { Patterson } \\
\text { Ringwald et al. (1984); Hawkins et al. } \\
\text { Winter \& Sion (2003); Sproats et al. }(1990) \\
\text { (1996); Tappert et al. }\end{array}$ \\
\hline
\end{tabular}




\section{Table 7-Continued}

\begin{tabular}{l}
\hline \hline $\begin{array}{l}\text { System References } \\
\text { Name }\end{array}$ \\
\hline
\end{tabular}

Note: Some systems (e.g. SS Cyg) have many more references and we list here only a few of the most relevant 

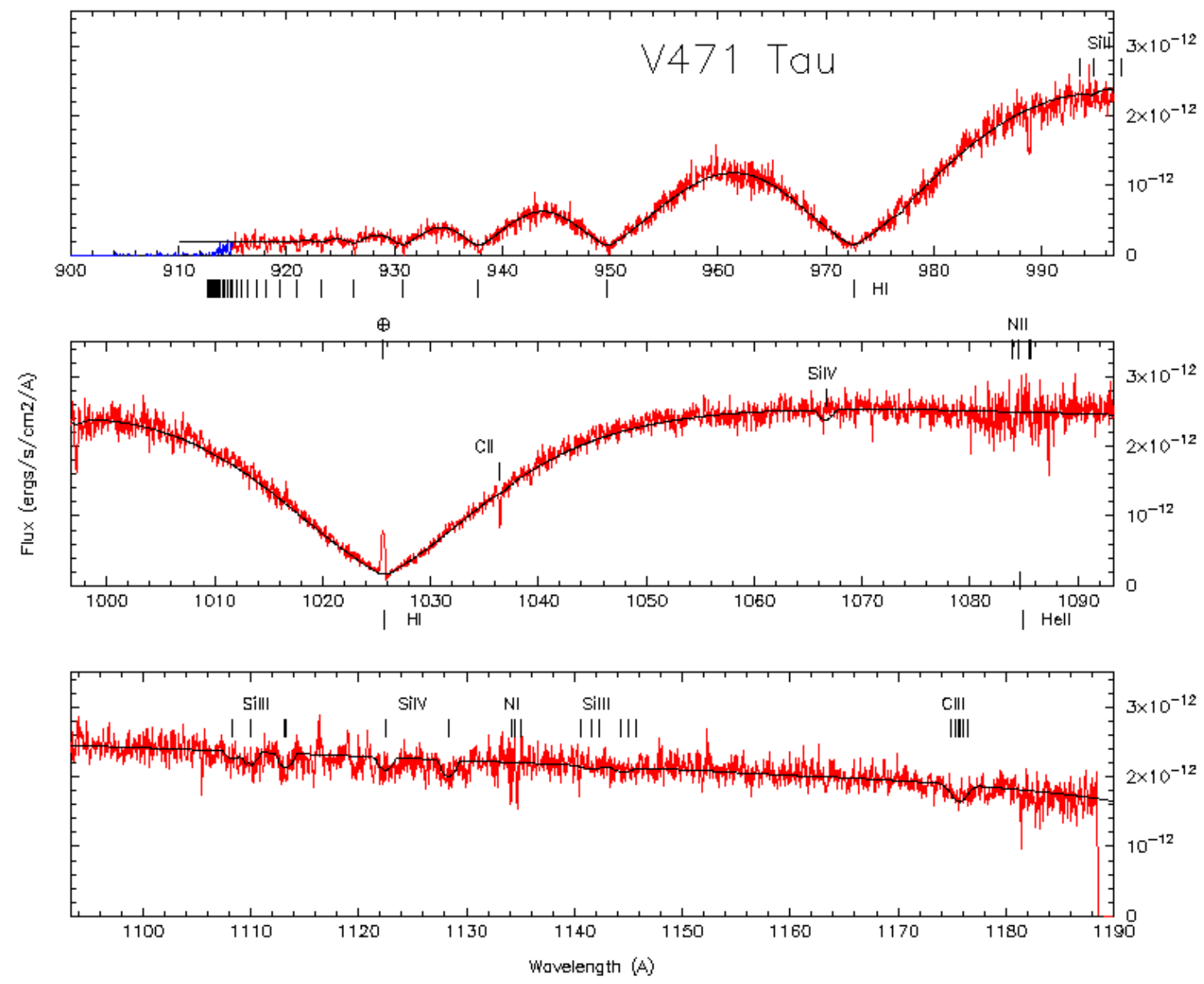

Fig. 1. - The FUSE spectrum of the WD in V471 Tau (in red) is fitted with a WD atmosphere model (in black). The WD atmosphere model is composed mainly of hydrogen and the Lyman series (marked below each panel) is clearly seen. The WD model has a gravity $\log (g)=8.3$, a temperature of $33,400 \mathrm{~K}$, a carbon abundances of $3 \times 10^{-4}$ solar, and a silicon abundance of $1.5 \times 10^{-2}$ solar. The rotational velocity used to match the shape of the lines is $250 \mathrm{~km} \mathrm{~s}^{-1}$ which is about a factor of four larger than expected. It is possible that the broadening of the lines is due to a weak Zeeman effect. This is a good example of an almost pure Hydrogen stellar atmosphere spectrum in which the main features are due to the absorption lines of the Hydrogen Lyman series. 

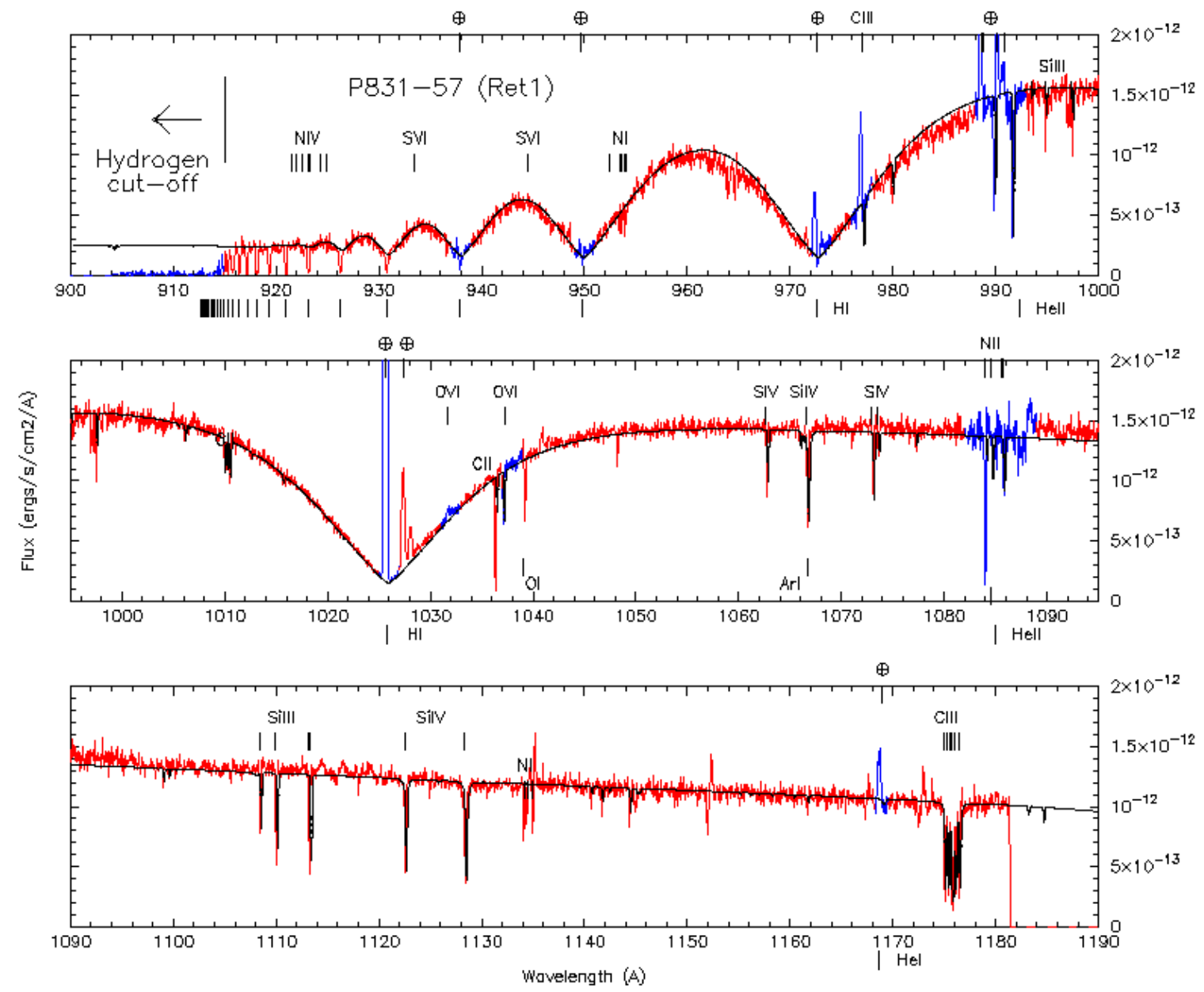

Fig. 2. - The FUSE spectrum of the wind accreting WD in P831-57 (in red) is fitted with a WD atmosphere model (in black). Regions that have been masked before the fitting are in blue. The WD model has a gravity of $\log (g)=7.85$, a temperature of $37,500 \mathrm{~K}$, carbon, sulfur, and nitrogen abundances of $1 \%$ solar, and silicon abundance of $2 \%$ solar. The stellar rotational broadening has been set to only a few $\mathrm{km}^{-1}$ in order to fit the sharp absorption lines. Airglow emissions are marked with a cross inside a circle. Compared to Fig.1, one can see how a slight increase in chemical abundances affects a WD stellar spectrum. 

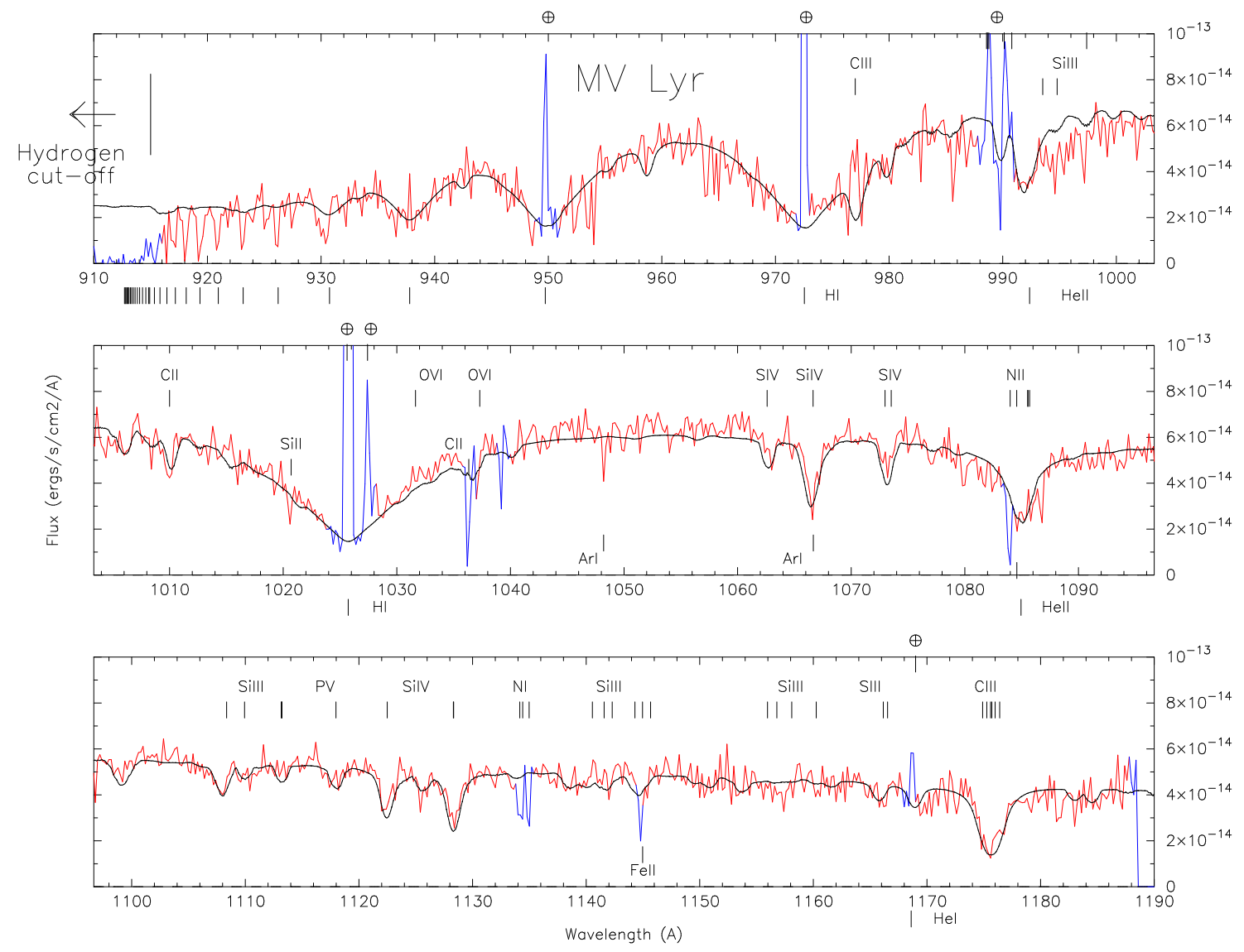

Fig. 3.- The FUSE spectrum of the Nova-like MV Lyr. This spectrum was obtained when MV Lyr dropped into a low state, therefore revealing the WD. The WD theoretical model has a surface gravity of $\log (g)=8.01$, a surface temperature $T_{\text {eff }}=45,000 \mathrm{~K}$, solar composition, and a rotational velocity of $200 \mathrm{~km} \mathrm{~s}^{-1}$. The model fit gives a distance of $500 \mathrm{pc}$. These results are in good agreement with the modeling of Hoard et al. (2004). This is a good example of a WD stellar atmosphere with solar composition and a moderate stellar rotation where the (metal) absorption lines significantly affect the continuum. 

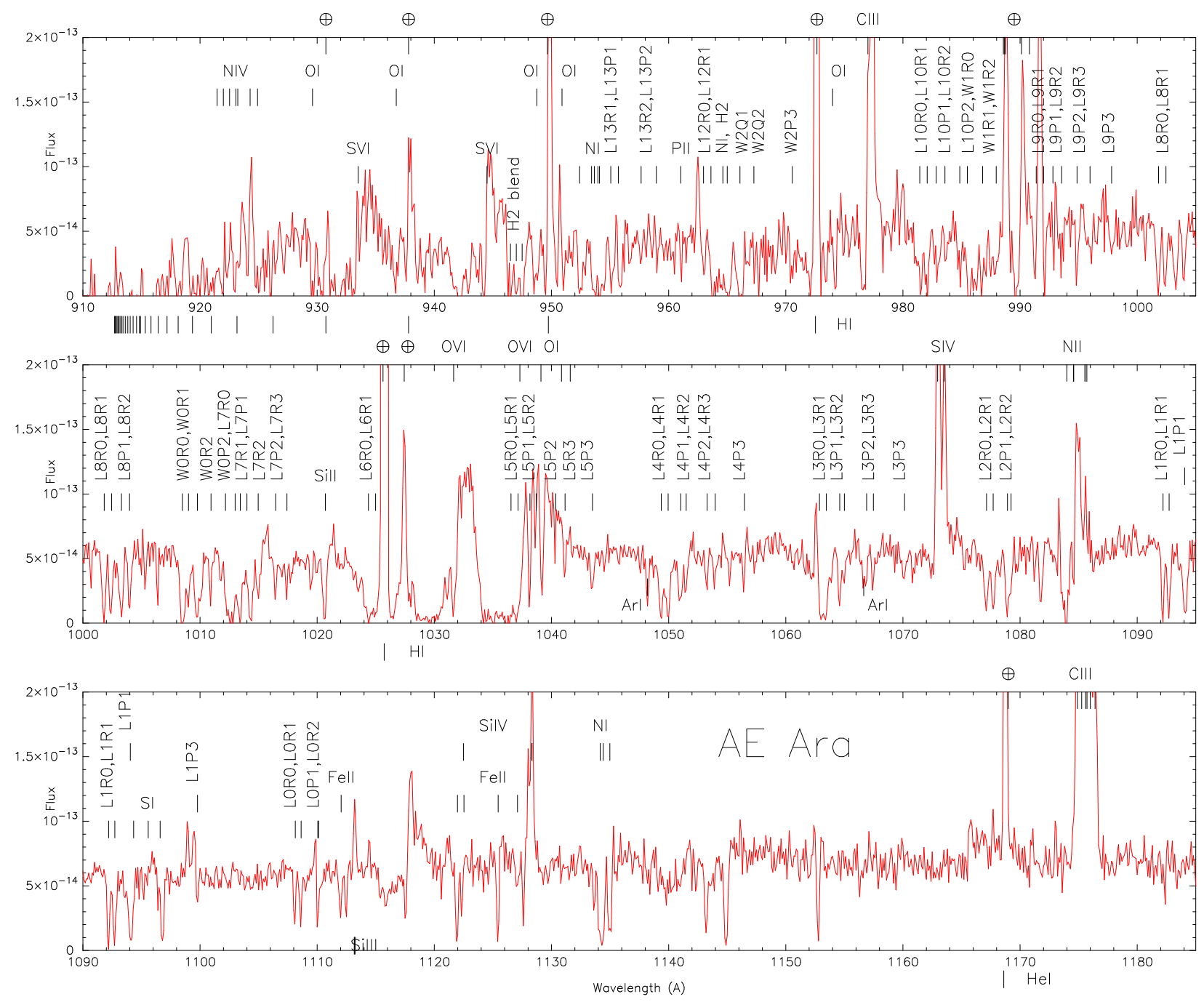

Fig. 4.- The FUSE spectrum of the symbiotic star AE Ara illustrates the multitude of ISM absorption lines that basically slice the spectrum starting around $1100 \AA$ all the way to the shortest wavelengths. The most prominent lines are identified by their band (Werner/W or Lyman/L), upper vibrational level (1-13), and rotational transition $(R, P$, or $Q$ with lower rotational state $J=1-3$ ). Additional ISM metal lines seen in the FUSE spectra of our catalog include lines from Ar I, Fe II, Ni, O I, P II, Si II, S III, C II, many of which are not present in the above spectrum of AE Ara. 

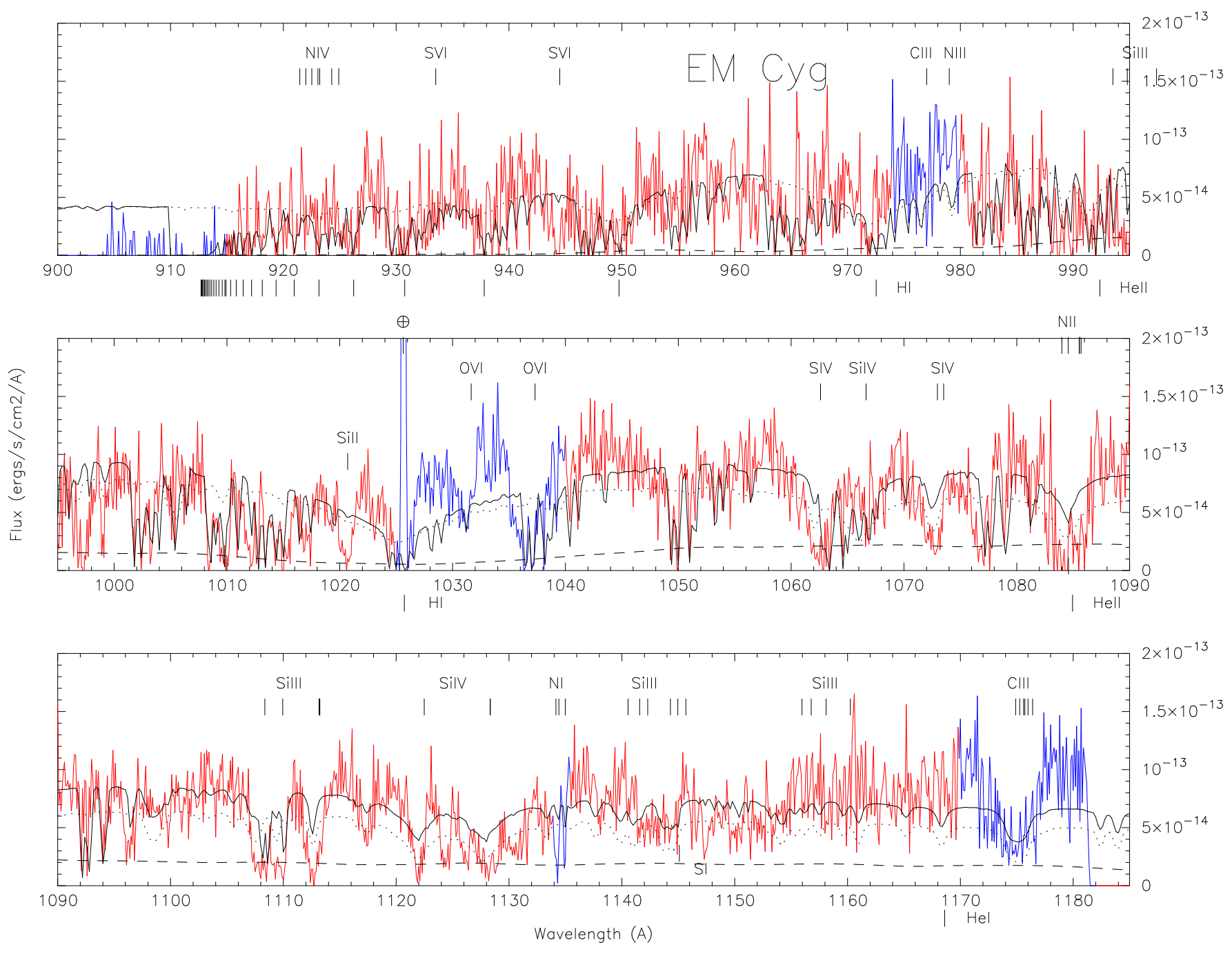

Fig. 5.- The FUSE spectrum (exposure 1) of the EM Cyg is shown with a WD+disk model fit (solid black line) including ISM absorption modeling. The $1 M_{\odot}$ WD model has a temperature of $50,000 \mathrm{~K}$, a projected rotational velocity of $100 \mathrm{~km} \mathrm{~s} s^{-1}$, over-solar abundances of Si (30), S(10) and $\mathrm{N}(10)$. The disk model has $\dot{M}=1 \times 10^{-10} M_{\odot} /$ yr with an inclination of $60^{\circ}$. The WD (dotted line) contributes $93 \%$ of the flux and the disk (dashed line) contributes the remaining $7 \%$. 

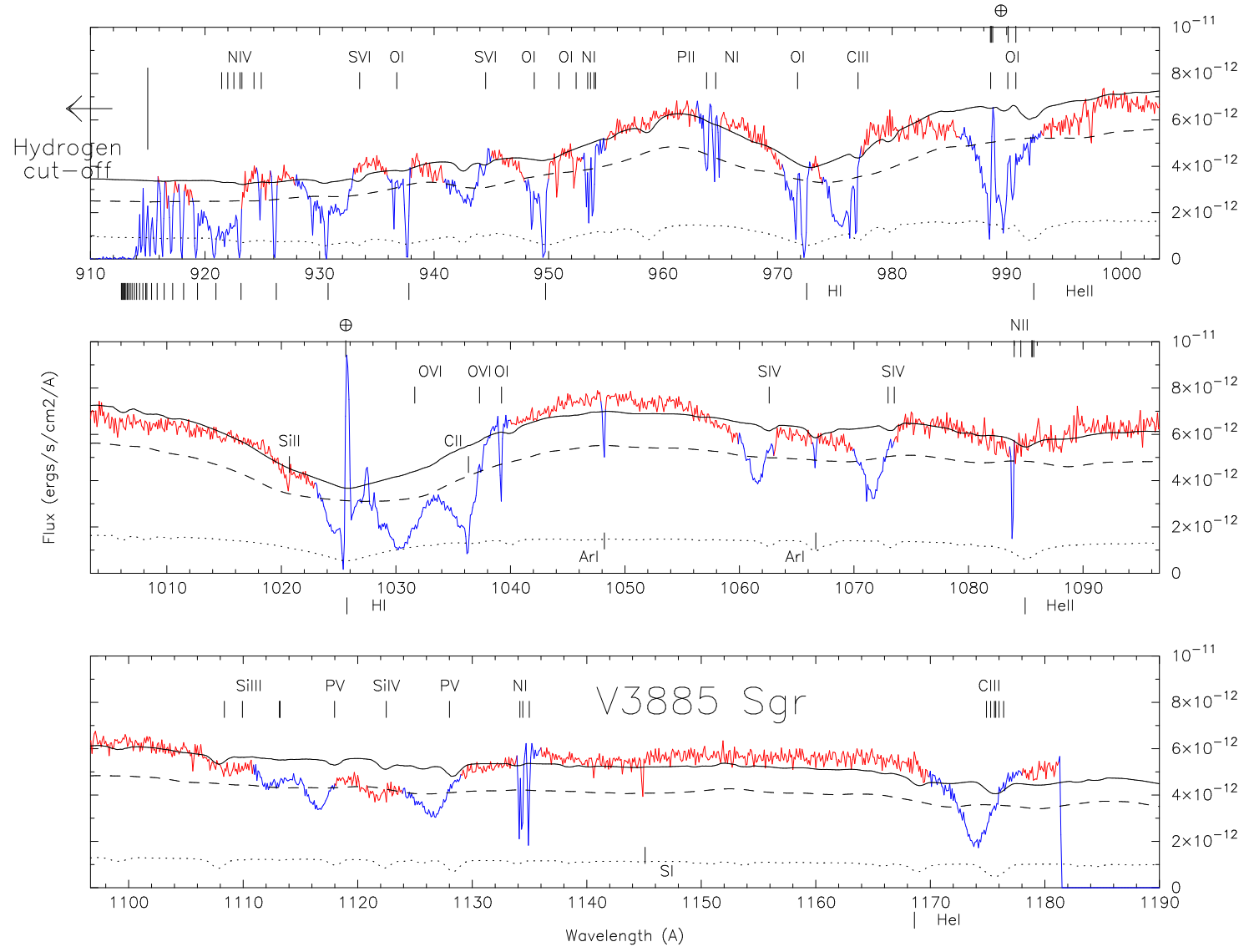

Fig. 6.- The FUSE spectrum of the nova-like V3885 Sgr is shown with a WD+disk model fit (solid black line) including the contribution from a hot boundary layer. The $0.7 M_{\odot} \mathrm{WD}$ model has a temperature of $60,000 \mathrm{~K}$, a projected rotational velocity of $200 \mathrm{~km} \mathrm{~s}$, solar abundances. The disk model has $\dot{M}=3 \times 10^{-9} M_{\odot} /$ yr with an inclination of $60^{\circ}$ and includes two inner (boundary layer) rings with $\mathrm{T}=175,000 \mathrm{~K}$. The WD (dotted line) contributes only $22 \%$ of the flux and the disk+BL (dashed line) contributes $78 \%$. 